\title{
Analysis of pseudoscalar and scalar $D$ mesons and charmonium decay width in hot magnetized asymmetric nuclear matter
}

\author{
Rajesh Kumar* and Arvind Kumar ${ }^{\dagger}$ \\ Department of Physics, Dr. B. R. Ambedkar National Institute of Technology Jalandhar, Jalandhar 144011 Punjab, India
}

(Received 27 August 2019; revised manuscript received 19 November 2019; published 8 January 2020)

\begin{abstract}
In this paper, we calculate the mass shift and decay constant of isospin-averaged pseudoscalar $\left(D^{+}, D^{0}\right)$ and scalar $\left(D_{0}^{+}, D_{0}^{0}\right)$ mesons by the magnetic-field-induced quark and gluon condensates at finite density and temperature of asymmetric nuclear matter. We have calculated the in-medium chiral condensates from the chiral SU(3) mean-field model and subsequently used these condensates in QCD sum rules to calculate the effective mass and decay constant of $D$ mesons. Consideration of external magnetic-field effects in hot and dense nuclear matter lead to appreciable modification in the masses and decay constants of $D$ mesons. Furthermore, we also studied the effective decay width of higher charmonium states $\left[\psi(3686), \psi(3770), \chi_{c 0}(3414), \chi_{c 2}(3556)\right]$ as a by-product by using the ${ }^{3} P_{0}$ model, which can have an important impact on the yield of $J / \psi$ mesons. The results of the present work will be helpful to understand the experimental observables of heavy-ion colliders which aim to produce matter at finite density and moderate temperature.
\end{abstract}

DOI: 10.1103/PhysRevC.101.015202

\section{INTRODUCTION}

Future heavy-ion colliders (HIC), such as the Japan Proton Accelerator Research Complex (J-PARC Japan), Compressed Baryonic Matter (CBM, GSI Germany), Proton AntiProton Annihilation in Darmstadt (PANDA, GSI Germany), and Nuclotron-based Ion Collider Facility (NICA, Dubna Russia), will shed light on the nonperturbative regime of the QCD by exploring the hadronic matter in a high-density and moderatetemperature range [1]. In HICs, two heavy-ion beams are smashed against each other and as a by-product quark gluon plasma (QGP) comes into existence under extreme conditions of temperature and density, but it lives for a very short interval of time [2]. Subsequently, with the decrease of temperature, a phase transition occurs in which the QGP is modified into hadronic matter by a process called hadronization [2]. Alongside the medium attributes such as isospin asymmetry (due to unequal number of protons and neutrons in heavy ion), strangeness (due to the presence of strange particles in the medium), temperature, and density, recently it was found that in HICs, a strong magnetic field is also produced having field strength of approximately $e B=2-15 m_{\pi}^{2}\left(1 m_{\pi}^{2}=2.818 \times\right.$ $10^{18}$ gauss) [3-5]. Since then, physicists have been trying to understand how the presence of this magnetic field affects the first- and second-order phase transitions [4,6-9]. The time duration for which the magnetic field remains is a very

\footnotetext{
*rajesh.sism@gmail.com

†iitd.arvind@gmail.com; kumara@nitj.ac.in
}

Published by the American Physical Society under the terms of the Creative Commons Attribution 4.0 International license. Further distribution of this work must maintain attribution to the author(s) and the published article's title, journal citation, and DOI. Funded by $S C O A P^{3}$. debateable topic. Many theories suggest that the magnetic field produced in HICs does not die immediately due to the interaction of itself with the medium. The primary magnetic field induces electric current in the matter and, due to Lenz's law, a secondary magnetic field comes into the picture which slows down the decay rate of the magnetic field [9-16]. These interactions increase the electric conductivity of the medium which further affects the relaxation time of the magnetic interaction, and this phenomenon is called the chiral magnetic effect $[4,6-8]$. The presence of the magnetic field affects the yield of in-medium/vacuum chiral condensates, and hence the location of the critical temperature $T_{c}$ is also affected and this process is known as (inverse) magnetic catalysis [6].

Near the hadron phase transition, it is not possible to detect QGP directly due to its short-lived nature, and hence many other indirect observations are used as a tool to understand its existence namely jet quenching [17], strangeness enhancements [18,19], dilepton enhancements [20-22], and $J / \psi(\Upsilon)$ suppression [23]. In 1986, Matsui and Satz proposed the idea of $J / \psi$ suppression on the basis of color Debye screening [23]. In this mechanism, when the Debye screening radius becomes less than the charm quark system's binding radius, the charm binding force can no longer keep the $c$ and $\bar{c}$ quarks together. These free charm quarks (antiquarks) form a bound state with free light quarks $(u, d, s)$ in the medium to form $D$ mesons. The in-medium effects on open charm mesons are more than the quarkonium. This is due to the fact that the in-medium properties of $D$ mesons depend on light quark condensates which varies appreciably with the medium, whereas for charmonia (bottomonia), it depends on gluon condensates which do not change much with density $[9,24]$. It may also be noted that $J / \psi$ suppression occurs not only due to QGP formation but also because of density-dependent suppression, comover scattering [25], and nuclear dependence of $D$ and $B$ (for $\Upsilon$ suppression) mesons [26,27]. Higher 
bottomonium and charmonium states decay to $\Upsilon$ and $J / \psi$ mesons, respectively, and hence are considered to be the major source of these ground-state mesons [23,28]. Under the effect of different medium conditions, if the mass of the $D(B)$ meson decreases appreciably, then these higher quarkonium states will prefer to decay in $D \bar{D}(B \bar{B})$ meson pairs rather than in conventional $J / \psi(\Upsilon)$ mesons. In $A A$ and $p \bar{A}$ collisions, the decay width of higher quarkonium states and other experimental observables [29] can be directly measured experimentally to validate the phenomenological results [30].

The QCD phase diagram is a graphical representation to account for the different QCD regime's physics with different medium parameters. To study this diagram in the hadron phase, several potential models are constructed on the basis of effective-field theory by incorporating basic properties of QCD, notably broken scale invariance and symmetry breaking [9,31]. Some of these models include the Walecka model [32], the Nambu-Jona-Lasinio (NJL) model [33], the chiral SU(3) model [9,31,34-36], QCD sum rules (QCDSR) [37-42], the quark-meson coupling (QMC) model [43-48], and the coupled-channels approach [49-52]. In the above approaches, the effect of thermal and quantum fluctuations are neglected by using mean-field approximations. These fluctuations are included by modified potential models such as the Polyakov quark meson (PQM) model [53,54], the Polyakov loop extended NJL (PNJL) model [55-57], and functional remormalization group (FRG) $[58,59]$ techniques. In addition, the decay width of the heavy mesons has been explained through various models, i.e., the ${ }^{3} S_{1}$ model [60], the elementary meson-emission model [61], the flux-tube model [62], and the ${ }^{3} P_{0}$ model [30].

Our present work presents three main points. First, we calculate the in-medium quark and gluon condensates from the chiral SU(3) mean-field model and, second, use them in QCDSR to calculate the medium-induced mass and decay constant of $D$ mesons in the presence of magnetic field. Last, by using the ${ }^{3} P_{0}$ model, we study the magnetic-field-induced decay width of higher charmonium states. The in-medium properties of meson under the effect of strong magnetic fields have been studied by various nonperturbative techniques in the literature [28,63-65]. For example, in Ref. [15], the properties of $D$ meson in strongly magnetized asymmetric nuclear matter was studied using the chiral SU(4) model and an additional positive mass shift for the charged $D$ meson, due to interaction with the magnetic field, was observed. In addition, under the effect of magnetic field the mass spectra of $D$ mesons and mixing effects between pseudoscalar and vector $D$ mesons have been studied with the use of operator product expansion technique of QCDSR by Gubler et al. [66]. The magnetic-induced decay width of higher charmonium states into lower charmonium states are calculated with the joint approach of the chiral model and the ${ }^{3} P_{0}$ model [67]. In this work, the author observed appreciable magnetic-field effects in cold nuclear matter. Processes such as chiral magnetic effect and (inverse) magnetic catalysis show great effect on the physics of deconfinement and chiral symmetry breaking. The analytic crossover, critical point, and phase transition of the QCD phase diagram is studied extensively in the literature $[6,68,69]$. In addition to these articles, the effect of strong magnetic fields is also studied on the properties of $\rho$ meson [70], $B$ meson [71,72], charmonium [9,16,73,74], and bottomonium states [74,75]. A lot of work has also been done without taking into account the effect of the magnetic field. For example, Tolos et al. investigated the increase in the mass of $D$ mesons in the nuclear medium using a coupledchannels approach [51]. In the QMC model, Tsushima and Khanna observed a negative shift of $D$ mesons in the nuclear medium and also discussed the possibility of the formation of $D$ mesic nuclei due to the attractive interaction of $D$ meson with the medium constituents [76]. The chiral SU(3) model was generalized to the $\mathrm{SU}(4)$ sector to study the in-medium mass of pseudoscalar $D$ mesons [77]. In this article, along with the in-medium mass of $D$ meson, authors also studied the decay width of higher charmonium states into $D \bar{D}$ pairs using ${ }^{3} P_{0}$ model. Using QCD sum rules, Wang et al. calculated the mass and decay constant of pseudoscalar, scalar, vector, and axial vector $D$ mesons by taking the contributions from nextto-leading-order terms [63]. Using QCDSR, the contribution up to the leading-order term has also been used to calculate the properties of scalar $D$ mesons [38]. By using the unification of the chiral SU(3) model and QCDSR, the in-medium mass and decay constants of pseudoscalar, scalar, vector, and axial vector $D$ mesons are calculated in the strange hadronic medium and a negative (scalar and vector) shift and a positive shift (pseudoscalar and axial vector) in the mass of $D$ mesons were observed [24,28,65,78]. In these articles, authors have also calculated the in-medium decay width of higher charmonium states [28] and scalar $D$ mesons [24]. The in-medium decay width of different heavy charmonia is also calculated using quark antiquark pair ${ }^{3} P_{0}$ model [30] and recently this model was also used to calculate the decay width of $\psi(4260)$ [79].

The outline of the present paper is as follows: In Secs. II A and IIB, we will briefly explain the formalism to calculate the effective masses and decay constant of pseudoscalar and scalar $D$ mesons under the effect of magnetic field. In Sec. II C, we will describe the methodology to calculate the decay width of higher charmonium states. In Sec. III, we will discuss the quantitative results of the present work and in Sec. IV, we will give a conclusion.

\section{FORMALISM}

We use the unification of the chiral SU(3) model and QCDSR techniques to study the effective mass and decay constants of scalar and pseudoscalar $D$ mesons. These nonperturbative techniques are constructed to understand the lowenergy QCD by using renormalization methods $[31,36,38,40]$. In this section, we gradually discuss the quark and gluon condensates, in-medium mass and decay constants of $D$ mesons, and the magnetic-field-induced charmonium decay width.

\section{A. Quark and gluon condensates from the chiral SU(3) model}

We use the nonperturbative chiral SU(3) model, constructed on the basis of effective-field theory. This model incorporates the basic QCD features such as nonlinear realization of chiral symmetry and trace anomaly $[9,31,36,80-$ 83]. In this framework, the trace anomaly (broken scale invariance) property of QCD is preserved by the introduction 
of scalar dilaton field $\chi[9,31]$. Also, the isospin asymmetry of the medium is incorporated by the introduction of scalar isovector delta field $\delta$ and vector-isovector field $\rho$ [36]. The model is built on the assumption of the mean-field potential, in which the mixing of vector and pseudoscalar mesons has been neglected, and hence the effect of thermal and quantum fluctuations are not studied in the present work
$[15,36]$. The effect of the external magnetic field is taken by adding the Lagrangian density due to magnetic field in the chiral effective Lagrangian density $[9,15]$. By minimizing the thermodynamic potential of the chiral SU(3) model $[9,74]$, the coupled equations of motion of the scalar $(\sigma, \zeta, \delta, \chi)$, and vector $(\omega, \rho)$, meson exchange fields are derived and are given as

$$
\begin{aligned}
& k_{0} \chi^{2} \sigma-4 k_{1}\left(\sigma^{2}+\zeta^{2}+\delta^{2}\right) \sigma-2 k_{2}\left(\sigma^{3}+3 \sigma \delta^{2}\right)-2 k_{3} \chi \sigma \zeta-\frac{d}{3} \chi^{4}\left(\frac{2 \sigma}{\sigma^{2}-\delta^{2}}\right)+\left(\frac{\chi}{\chi_{0}}\right)^{2} m_{\pi}^{2} f_{\pi}=\sum g_{\sigma i} \rho_{i}^{s}, \\
& k_{0} \chi^{2} \zeta-4 k_{1}\left(\sigma^{2}+\zeta^{2}+\delta^{2}\right) \zeta-4 k_{2} \zeta^{3}-k_{3} \chi\left(\sigma^{2}-\delta^{2}\right)-\frac{d}{3} \frac{\chi^{4}}{\zeta}+\left(\frac{\chi}{\chi_{0}}\right)^{2}\left[\sqrt{2} m_{K}^{2} f_{K}-\frac{1}{\sqrt{2}} m_{\pi}^{2} f_{\pi}\right]=\sum g_{\zeta i} \rho_{i}^{s}, \\
& k_{0} \chi^{2} \delta-4 k_{1}\left(\sigma^{2}+\zeta^{2}+\delta^{2}\right) \delta-2 k_{2}\left(\delta^{3}+3 \sigma^{2} \delta\right)+2 k_{3} \chi \delta \zeta+\frac{2}{3} d \chi^{4}\left(\frac{\delta}{\sigma^{2}-\delta^{2}}\right)=\sum g_{\delta i} \tau_{3} \rho_{i}^{s}, \\
& k_{0} \chi\left(\sigma^{2}+\zeta^{2}+\delta^{2}\right)-k_{3}\left(\sigma^{2}-\delta^{2}\right) \zeta+\chi^{3}\left[1+\ln \left(\frac{\chi^{4}}{\chi_{0}^{4}}\right)\right]+\left(4 k_{4}-d\right) \chi^{3}-\frac{4}{3} d \chi^{3} \ln \left\{\left[\frac{\left(\sigma^{2}-\delta^{2}\right) \zeta}{\sigma_{0}^{2} \zeta_{0}}\right]\left(\frac{\chi}{\chi_{0}}\right)^{3}\right\} \\
& \quad+\frac{2 \chi}{\chi_{0}^{2}}\left[m_{\pi}^{2} f_{\pi} \sigma+\left(\sqrt{2} m_{K}^{2} f_{K}-\frac{1}{\sqrt{2}} m_{\pi}^{2} f_{\pi}\right) \zeta\right]-\frac{\chi}{\chi_{0}^{2}}\left(m_{\omega}^{2} \omega^{2}+m_{\rho}^{2} \rho^{2}\right)=0, \\
& \left(\frac{\chi}{\chi_{0}}\right)^{2} m_{\omega}^{2} \omega+g_{4}\left(4 \omega^{3}+12 \rho^{2} \omega\right)=\sum g_{\omega i} \rho_{i}^{v},
\end{aligned}
$$

and

$$
\left(\frac{\chi}{\chi_{0}}\right)^{2} m_{\rho}^{2} \rho+g_{4}\left(4 \rho^{3}+12 \omega^{2} \rho\right)=\sum g_{\rho i} \tau_{3} \rho_{i}^{v}
$$

respectively.

In the above, the parameters $k_{0}, k_{2}$, and $k_{4}$ are fitted to reproduce the vacuum values of scalar meson fields, and the other parameters, such as $k_{1}$, are constrained to obtain the in-medium mass of nucleon at nuclear saturation density, $\rho_{N}$, and the parameter $k_{3}$ is selected to generate the masses of $\eta$ and $\eta^{\prime}$ mesons. In addition, the parameters $f_{\pi}, f_{K}$ and $m_{\pi}, m_{K}$ are the decay constants and masses of pions and kaons, respectively. Moreover, the effect of isospin asymmetry is introduced in the nuclear matter calculations by the parameter $\left(\eta=-\frac{\Sigma_{i} \tau_{3 i} \rho_{i}^{v}}{2 \rho_{N}}\right)$, where $\rho_{i}^{s}$ and $\rho_{i}^{v}$ represent the scalar and vector densities of the $i$ th nucleon $(i=n, p)$ in the presence of magnetic field which is applied in the $z$ direction $[9,84,85]$ and $\tau_{3 i}$ is the $I_{3}$ component of isospin. With the interaction of protons with magnetic field, Landau quantization takes place [9,84]. This circular confined motion disrupts the net momentum $k$ in two parts, i.e., $k_{\perp}$ (perpendicular to the $z$ axis) and $k_{\|}$(parallel to $z$ axis) [9].

The magnetic-field-induced scalar density as well as the vector density of uncharged neutron given in Eqs. (1) and (5) are given as $[84,85]$

$$
\rho_{n}^{s}=\frac{1}{2 \pi^{2}} \sum_{s= \pm 1} \int_{0}^{\infty} k_{\perp}^{n} d k_{\perp}^{n}\left[1-\frac{s \mu_{N} \kappa_{n} B}{\sqrt{m_{n}^{* 2}+\left(k_{\perp}^{n}\right)^{2}}}\right] \int_{0}^{\infty} d k_{\|}^{n} \frac{m_{n}^{*}}{\tilde{E}_{s}^{n}}\left(f_{k, s}^{n}+\bar{f}_{k, s}^{n}\right)
$$

and

$$
\rho_{n}^{v}=\frac{1}{2 \pi^{2}} \sum_{s= \pm 1} \int_{0}^{\infty} k_{\perp}^{n} d k_{\perp}^{n} \int_{0}^{\infty} d k_{\|}^{n}\left(f_{k, s}^{n}-\bar{f}_{k, s}^{n}\right)
$$

respectively. Similarly, for the charged proton, the scalar and vector densities are given by [84,85]

$$
\rho_{p}^{s}=\frac{\left|q_{p}\right| B m_{p}^{*}}{2 \pi^{2}}\left[\sum_{\nu=0}^{\nu_{\max }^{(s=1)}} \int_{0}^{\infty} \frac{d k_{\|}^{p}}{\sqrt{\left(k_{\|}^{p}\right)^{2}+\left(\bar{m}_{p}\right)^{2}}}\left(f_{k, v, s}^{p}+\bar{f}_{k, v, s}^{p}\right)+\sum_{\nu=1}^{\nu_{\max }^{(s=-1)}} \int_{0}^{\infty} \frac{d k_{\|}^{p}}{\sqrt{\left(k_{\|}^{p}\right)^{2}+\left(\bar{m}_{p}\right)^{2}}}\left(f_{k, v, s}^{p}+\bar{f}_{k, v, s}^{p}\right)\right]
$$

and

$$
\rho_{p}^{v}=\frac{\left|q_{p}\right| B}{2 \pi^{2}}\left[\sum_{\nu=0}^{\nu_{\text {max }}^{(s=1)}} \int_{0}^{\infty} d k_{\|}^{p}\left(f_{k, \nu, s}^{p}-\bar{f}_{k, \nu, s}^{p}\right)+\sum_{\nu=1}^{\nu_{\text {max }}^{(s=-1)}} \int_{0}^{\infty} d k_{\|}^{p}\left(f_{k, \nu, s}^{p}-\bar{f}_{k, \nu, s}^{p}\right)\right]
$$


respectively, where $\bar{m}_{p}$ is the induced mass under the effect of magnetic field, which is defined as

$$
\bar{m}_{p}=\sqrt{m_{p}^{* 2}+2 \nu\left|q_{p}\right| B}-s \mu_{N} \kappa_{p} B
$$

In the above equations, $v$ represents the Landau quantized levels and $m_{i}^{*}=-\left(g_{\sigma i} \sigma+g_{\zeta i} \zeta+g_{\delta i} \tau_{3 i} \delta\right)$ is the effective mass of the nucleons. Here $g_{\sigma i}, g_{\zeta i}$, and $g_{\delta i}$ represent the coupling constants of $i$ th nucleons with $\sigma, \zeta$, and $\delta$ fields, respectively. The effective single-particle energy of a proton is given by $\tilde{E}_{v, s}^{p}=\sqrt{\left(k_{\|}^{p}\right)^{2}+\left(\sqrt{m_{p}^{* 2}+2 \nu\left|q_{p}\right| B}-s \mu_{N} \kappa_{p} B\right)^{2}}$, whereas for a neutron its expression is given as $\tilde{E}_{s}^{n}=\sqrt{\left(k_{\|}^{n}\right)^{2}+\left[\sqrt{m_{n}^{* 2}+\left(k_{\perp}^{n}\right)^{2}}-s \mu_{N} \kappa_{n} B\right]^{2}}$. The constants $k_{i}$ and $s$ are the anomalous magnetic moment and the spin of the nucleons, respectively. In addition, $f_{k, v, s}^{n}, \bar{f}_{k, v, s}^{n}, f_{k, s}^{p}$, and $\bar{f}_{k, s}^{p}$ represent the finite-temperature distribution functions for neutrons and protons and their antiparticles and are given as

$$
\begin{aligned}
f_{k, s}^{n} & =\frac{1}{1+\exp \left[\beta\left(\tilde{E}_{s}^{n}-\mu_{n}^{*}\right)\right]} \\
\bar{f}_{k, s}^{n} & =\frac{1}{1+\exp \left[\beta\left(\tilde{E}_{s}^{n}+\mu_{n}^{*}\right)\right]} \\
f_{k, v, s}^{p} & =\frac{1}{1+\exp \left[\beta\left(\tilde{E}_{v, s}^{p}-\mu_{p}^{*}\right)\right]} \\
\bar{f}_{k, v, s}^{p} & =\frac{1}{1+\exp \left[\beta\left(\tilde{E}_{v, s}^{p}+\mu_{p}^{*}\right)\right]} .
\end{aligned}
$$

As we will see, to calculate the effective mass and decay constant of scalar and pseudoscalar $D$ mesons using QCDSR, we need the values of quarks and gluon condensates. In the chiral model, the scalar quark condensates can be related to symmetry breaking via the following relation [9]:

$$
\sum_{i} m_{i}\left\langle\bar{q}_{i} q_{i}\right\rangle_{\rho_{N}}=-\mathcal{L}_{\mathrm{SB}}
$$

where $\mathcal{L}_{\mathrm{SB}}$ is an explicit symmetry-breaking Lagrangian term [9], and by using this equation we formulated the up- and down-quark condensates, which are expressed as

$$
\langle\bar{u} u\rangle_{\rho_{N}}=\frac{1}{m_{u}}\left(\frac{\chi}{\chi_{0}}\right)^{2}\left[\frac{1}{2} m_{\pi}^{2} f_{\pi}(\sigma+\delta)\right]
$$

and

$$
\langle\bar{d} d\rangle_{\rho_{N}}=\frac{1}{m_{d}}\left(\frac{\chi}{\chi_{0}}\right)^{2}\left[\frac{1}{2} m_{\pi}^{2} f_{\pi}(\sigma-\delta)\right],
$$

respectively. In the above $m_{u}$ and $m_{d}$ are the masses of up-quark and down-quark, respectively. Also, by using the broken scale invariance property of QCD $[9,31,36]$, the scalar gluon condensate $G_{\rho_{N}}=\left\langle\frac{\alpha_{s}}{\pi} G_{\mu \nu}^{a} G^{a \mu \nu}\right\rangle_{\rho_{N}}$ is formulated by the comparison of energy-momentum tensor (EMT) of QCD with the EMT of the chiral model and is expressed in terms of scalar fields through the relation [9]

$$
\begin{aligned}
\left\langle\frac{\alpha_{s}}{\pi} G_{\mu \nu}^{a} G^{a \mu \nu}\right\rangle_{\rho_{N}}= & \frac{8}{9}\left\{(1-d) \chi^{4}+\left(\frac{\chi}{\chi_{0}}\right)^{2}\left[m_{\pi}^{2} f_{\pi} \sigma\right.\right. \\
& \left.\left.+\left(\sqrt{2} m_{K}^{2} f_{K}-\frac{1}{\sqrt{2}} m_{\pi}^{2} f_{\pi}\right) \zeta\right]\right\} .
\end{aligned}
$$

The value of $d=\frac{2}{11}$ has been taken from the QCD beta function, $\beta_{\mathrm{QCD}}$ at the one-loop level [31].

\section{B. Masses and decay constant of $D$ mesons from QCDSR}

In this subsection, we discuss the QCDSR to calculate the in-medium mass shift and decay constant of isospin averaged pseudoscalar $\left(D^{+}, D_{0}\right)$ and scalar $\left(D_{0}^{+}, D_{0}^{0}\right)$ mesons. QCDSR is a nonperturbative technique which is based on the Borel transformation and operator product expansion (OPE) method $[38,40,42,63]$. These methods are used to deal with the divergence that occurs in the asymptotic perturbative series $[38,40]$. We will see that the mass and decay constant of these open charm mesons is expressed in terms of scalar and gluon condensates, which contains the effect of medium parameters such as temperature, density, asymmetry, and magnetic field. We start with the two-point current correlation function $\Pi(q)$ which represents the Fourier transformation of the timeordered product of the isospin-averaged meson current, $J^{\prime}(x)$, and can be written as $[28,63]$

$$
\Pi(q)=i \int d^{4} x e^{i q \cdot x}\left\langle\mathcal{T}\left\{J^{\prime}(x) J^{\prime \dagger}(0)\right\}\right\rangle_{\rho_{N}, T},
$$

where $q$ is the four-momentum and $\rho_{N}$ and $T$ represent the nucleon density and temperature of the medium. In Refs. [39,86], the mass splitting of the different oppositely charged mesons are also investigated by dividing the current correlation function in even and odd terms. In this paper, we have considered the average meson currents of the particle $D$ and their antiparticle $\bar{D}$. The average current of scalar and pseudoscalar meson is given by the following mathematical relations:

$$
J(x)=J^{\dagger}(x)=\frac{\bar{c}(x) q(x)+\bar{q}(x) c(x)}{2}
$$

and

$$
J_{5}(x)=J_{5}^{\dagger}(x)=\frac{\bar{c}(x) i \gamma_{5} q(x)+\bar{q}(x) i \gamma_{5} c(x)}{2},
$$

respectively. In the above, the quark operator $q(x)$ is for $u$ and $d$ quarks and $c(x)$ is the charm quark operator. The selection of $q$ depends on the quark content of the given meson. From the quark composition of $D$ mesons one can easily understand that the $\left(D^{+}, D^{0}\right)$ and $\left(D_{0}^{+}, D_{0}^{0}\right)$ form the isospin doublets and they show mass splitting in the presence of isospin asymmetric medium [28]. Now, for the nuclear matter in the Fermi gas approximation, we divide the correlation function $\Pi(q)$ into vacuum, static nucleon, and thermal parts as

$$
\Pi(q)=\Pi_{0}(q)+\frac{\rho_{N}}{2 m_{N}} T_{N}(q)+\Pi_{\text {P.B. }}(q, T),
$$

where $T_{N}(q)$ is the forward-scattering amplitude and $m_{N}$ denotes the nucleon mass. The third term, i.e., the pion bath 
contribution, represents the thermal effects of the medium and is given as [87]

$$
\Pi_{\text {P.B. }}(q, T)=i \int d^{4} x e^{i q \cdot x}\left\langle\mathcal{T}\left\{J^{\prime}(x) J^{\prime \dagger}(0)\right\}\right\rangle_{T} .
$$

In the present investigation, we can neglect this thermal effects term, as the temperature and magnetic effects of the medium are incorporated by quark and gluon condensates, which are calculated in terms of the meson exchange fields as discussed under Sec. II A [24,78]. By neglecting the third term from Eq. (21), the expression becomes

$$
\Pi(q)=\Pi_{0}(q)+\frac{\rho_{N}}{2 m_{N}} T_{N}(q) .
$$

The forward-scattering amplitude $T_{N}(q)$ can be written as

$$
T_{N}(\omega, \boldsymbol{q})=i \int d^{4} x e^{i q \cdot x}\left\langle N(p)\left|T\left\{J(x) J^{\dagger}(0)\right\}\right| N(p)\right\rangle .
$$

The amplitude $T_{N}(\omega, \boldsymbol{q})$ can be related to the $D N\left(D_{0} N\right)$ scattering $T$ matrix in the limit of $\boldsymbol{q} \rightarrow \mathbf{0}$,

$$
\mathcal{T}_{D / D_{0} N}\left(m_{D / D_{0}}, 0\right)=8 \pi\left(m_{N}+m_{D / D_{0}}\right) a_{D / D_{0}},
$$

where $a_{D / D_{0}}$ is the scattering lengths of $D N\left(D_{0} N\right)$ interactions. This scattering matrix can be represented in terms of phenomenological spectral density $\rho(\omega, 0)$, which can be parametrized in three unknown parameters, $a, b$, and $c$ [38],

$$
\begin{aligned}
\rho(\omega, 0)= & -\frac{1}{\pi} \operatorname{Im}\left[\frac{\mathcal{T}_{D / D_{0} N}(\omega, \mathbf{0})}{\left(\omega^{2}-m_{D / D_{0}}^{2}+i \varepsilon\right)^{2}}\right] \frac{f_{D / D_{0}}^{2} m_{D / D_{0}}^{4}}{m_{c}^{2}}+\cdots, \\
= & a \frac{d}{d \omega^{2}} \delta\left(\omega^{2}-m_{D / D_{0}}^{2}\right)+b \delta\left(\omega^{2}-m_{D / D_{0}}^{2}\right) \\
& +c \delta\left(\omega^{2}-s_{0}\right) .
\end{aligned}
$$

In above equation, the first term represents the double pole term, which is related to the on-shell effect of $T$ matrices and can be related to the scattering length as

$$
a_{D / D_{0}}=\frac{a m_{c}^{2}}{f_{D / D_{0}}^{2} m_{D / D_{0}}^{4}\left[-8 \pi\left(m_{N}+m_{D / D_{0}}\right)\right]} .
$$

Furthermore, the second term in Eq. (26) represents the single pole term that relates the off-shell effects of $T$ matrices; the last term corresponds to the remaining contributions (continuum), where $s_{0}$ denotes the continuum threshold parameter.

The shift in the above-mentioned masses $m_{D / D_{0}}$ and decay constants $f_{D / D_{0}}$ of the open charm mesons can be written as [63]

$$
\Delta m_{D / D_{0}}^{*}=2 \pi \frac{m_{N}+m_{D / D_{0}}}{m_{N} m_{D / D_{0}}} \rho_{N} a_{D / D_{0}}
$$

and

$$
\Delta f_{D / D_{0}}^{*}=\frac{m_{c}^{2}}{2 f_{D / D_{0}} m_{D / D_{0}}^{4}}\left(\frac{b \rho_{N}}{2 m_{N}}-\frac{4 f_{D / D_{0}}^{2} m_{D / D_{0}}^{3} \Delta m_{D / D_{0}}}{m_{c}^{2}}\right),
$$

respectively. Hence the effective mass of open charm mesons can be written as

$$
m_{D / D_{0}}^{*}=m_{D / D_{0}}+\Delta m_{D / D_{0}}^{*} .
$$

Note that the effective mass $\left(m_{D / D_{0}}^{*}\right)$ is the total excitation energy of the particle at zero momentum in the medium and $m_{D / D_{0}}$ denotes a vacuum mass of pseudoscalar and scalar $D$ mesons.

As discussed earlier, the Landau quantization takes place with the interaction of charged particle with magnetic field. This interaction invokes an additional positive shift in the mass of the charged $D^{+}, D_{0}^{+}$meson and this lead to

$$
m_{D^{+} / D_{0}^{+}}^{* *}=\sqrt{m_{D^{+} / D_{0}^{+}}^{* 2}+|e B|} .
$$

On the other hand, due to their uncharged nature the neutral pseudoscalar $\left(D^{0}\right)$ and scalar $\left(D_{0}^{0}\right)$ mesons have no modification due to magnetic field.

We get the analytic QCDSR in terms of two unknown parameters $a$ and $b$ by equating the Borel-transformed forwardscattering amplitude $T_{N}(\omega, \boldsymbol{q})$ in the OPE side with the Boreltransformed forward-scattering amplitude $T_{N}(\omega, \boldsymbol{q})$ in the phenomenological side [38]. The parametrized QCDSR are given by equation

$$
a C_{a}+b C_{b}=C_{f} .
$$

The explicit form of Borel-transformed coefficients having next-to-leading-order contributions for the pseudoscalar current $J_{5}(x)$ is [63]

$$
\begin{aligned}
& C_{a}=\frac{1}{M^{2}} \exp \left(-\frac{m_{D}^{2}}{M^{2}}\right)-\frac{s_{0}}{m_{D}^{4}} \exp \left(-\frac{s_{0}}{M^{2}}\right), \\
& C_{b}=\exp \left(-\frac{m_{D}^{2}}{M^{2}}\right)-\frac{s_{0}}{m_{D}^{2}} \exp \left(-\frac{s_{0}}{M^{2}}\right),
\end{aligned}
$$

and

$$
\begin{aligned}
C_{f}= & \frac{2 m_{N}\left(m_{H}+m_{N}\right)}{\left(m_{H}+m_{N}\right)^{2}-m_{D}^{2}}\left(\frac{f_{D} m_{D}^{2} g_{\mathrm{DNH}}}{m_{c}}\right)^{2}\left\{\left[\frac{1}{M^{2}}-\frac{1}{m_{D}^{2}-\left(m_{H}+m_{N}\right)^{2}}\right] \exp \left(-\frac{m_{D}^{2}}{M^{2}}\right)\right. \\
& \left.+\frac{1}{\left(m_{H}+m_{N}\right)^{2}-m_{D}^{2}} \exp \left[-\frac{\left(m_{H}+m_{N}\right)^{2}}{M^{2}}\right]\right\}-\frac{m_{c}\langle\bar{q} q\rangle_{N}}{2}\left\{1+\frac{\alpha_{s}}{\pi}\left[6-\frac{4 m_{c}^{2}}{3 M^{2}}\right.\right. \\
& \left.\left.-\frac{2}{3}\left(1-\frac{m_{c}^{2}}{M^{2}}\right) \log \frac{m_{c}^{2}}{\mu^{2}}-2 \Gamma\left(0, \frac{m_{c}^{2}}{M^{2}}\right) \exp \left(\frac{m_{c}^{2}}{M^{2}}\right)\right]\right\} \exp \left(-\frac{m_{c}^{2}}{M^{2}}\right) \\
& +\frac{1}{2}\left\{-2\left(1-\frac{m_{c}^{2}}{M^{2}}\right)\left\langle q^{\dagger} i D q\right\rangle_{N}+\frac{4 m_{c}}{M^{2}}\left(1-\frac{m_{c}^{2}}{2 M^{2}}\right)\langle\bar{q} i D i D q\rangle_{N}+\frac{1}{12}\left\langle\frac{\alpha_{s} G G}{\pi}\right\rangle_{N}\right\} \exp \left(-\frac{m_{c}^{2}}{M^{2}}\right)
\end{aligned}
$$


For the scalar current $J(x)$, we have $[64,65]$

$$
C_{a}=\frac{1}{M^{2}} \exp \left(-\frac{m_{D_{0}}^{2}}{M^{2}}\right)-\frac{s_{0}}{m_{D_{0}}^{4}} \exp \left(-\frac{s_{0}}{M^{2}}\right), \quad C_{b}=\exp \left(-\frac{m_{D_{0}}^{2}}{M^{2}}\right)-\frac{s_{0}}{m_{D_{0}}^{2}} \exp \left(-\frac{s_{0}}{M^{2}}\right),
$$

and

$$
\begin{aligned}
C_{f}= & \frac{2 m_{N}\left(m_{H}-m_{N}\right)}{\left(m_{H}-m_{N}\right)^{2}-m_{D_{0}}^{2}}\left(\frac{f_{D_{0}} m_{D_{0}}^{2} g_{D_{0} N H}}{m_{c}}\right)^{2}\left\{\left[\frac{1}{M^{2}}-\frac{1}{m_{D_{0}}^{2}-\left(m_{H}-m_{N}\right)^{2}}\right] \exp \left(-\frac{m_{D_{0}}^{2}}{M^{2}}\right)\right. \\
& \left.+\frac{1}{\left(m_{H}-m_{N}\right)^{2}-m_{D_{0}}^{2}} \exp \left[-\frac{\left(m_{H}-m_{N}\right)^{2}}{M^{2}}\right]\right\}+\frac{m_{c}\langle\bar{q} q\rangle_{N}}{2} \exp \left(-\frac{m_{c}^{2}}{M^{2}}\right) \\
& +\frac{1}{2}\left\{-2\left(1-\frac{m_{c}^{2}}{M^{2}}\right)\left\langle q^{\dagger} i D q\right\rangle_{N}-\frac{4 m_{c}}{M^{2}}\left(1-\frac{m_{c}^{2}}{2 M^{2}}\right)\langle\bar{q} i D i D q\rangle_{N}\right\} \exp \left(-\frac{m_{c}^{2}}{M^{2}}\right) \\
& +\frac{1}{16}\left\langle\frac{\alpha_{s} G G}{\pi}\right\rangle_{N} \int_{0}^{1} d x\left(1+\frac{\widetilde{m}_{c}^{2}}{M^{2}}\right) \exp \left(-\frac{\widetilde{m}_{c}^{2}}{M^{2}}\right)-\frac{1}{48 M^{4}}\left\langle\frac{\alpha_{s} G G}{\pi}\right\rangle_{N} \int_{0}^{1} d x \frac{1-x}{x} \widetilde{m}_{c}^{4} \exp \left(-\frac{\widetilde{m}_{c}^{2}}{M^{2}}\right)
\end{aligned}
$$

In the above equations, $\frac{1}{M^{2}}$ is the Borel mass operator and $\langle\bar{q} q\rangle_{N},\left\langle q^{\dagger} i D q\right\rangle_{N},\langle\bar{q} i D i D q\rangle_{N}$, and $\left\langle\frac{\alpha_{s} G G}{\pi}\right\rangle_{N}$ are the nucleon expectation values of different quark and gluon condensates. Also, $\Gamma(0, x)=e^{-x} \int_{0}^{\infty} d t \frac{1}{t+x} e^{-t}$ and $\widetilde{m}_{c}^{2}=m_{c}^{2} / x$. We will see later that as compared to scalar quark condensates $\langle\bar{q} q\rangle_{N}$, the impact of other quark condensates (OQC) $\left\langle q^{\dagger} i D q\right\rangle_{N}$, $\langle\bar{q} i D i D q\rangle_{N}$ is very small on the observables of the $D$ mesons.

The nucleon expectation values of the chiral condensates can be calculated by using

$$
\begin{aligned}
\mathcal{O}_{\rho_{N}} & =\mathcal{O}_{\text {vac }}+4 \int \frac{d^{3} p}{(2 \pi)^{3} 2 E_{p}} n_{F}\langle N(p)|\mathcal{O}| N(p)\rangle \\
& =\mathcal{O}_{\text {vac }}+\frac{\rho_{N}}{2 m_{N}} \mathcal{O}_{N},
\end{aligned}
$$

where $\mathcal{O}$ denotes an operator. Now, by taking the expectation values at both sides of above equation,

$$
\begin{aligned}
\langle\mathcal{O}\rangle_{\rho_{N}} & =\langle\mathcal{O}\rangle_{\text {vac }}+\frac{\rho_{N}}{2 m_{N}}\langle\mathcal{O}\rangle_{N}, \\
\langle\mathcal{O}\rangle_{N} & =\frac{2 m_{N}}{\rho_{N}}\left(\langle\mathcal{O}\rangle_{\rho_{N}}-\langle\mathcal{O}\rangle_{\text {vac }}\right),
\end{aligned}
$$

where $\langle\mathcal{O}\rangle_{\text {vac }}$ and $\langle\mathcal{O}\rangle_{N}$ denote the vacuum operator and nuclear matter-induced operator in the Fermi gas model, respectively [88].

Following this, the nucleon expectation values of light quark and gluon condensates are expressed as

$$
\begin{aligned}
\langle\bar{u} u\rangle_{N} & =\left[\langle\bar{u} u\rangle_{\rho_{N}}-\langle\bar{u} u\rangle_{\mathrm{vac}}\right] \frac{2 m_{N}}{\rho_{N}}, \\
\langle\bar{d} d\rangle_{N} & =\left[\langle\bar{d} d\rangle_{\rho_{N}}-\langle\bar{d} d\rangle_{\mathrm{vac}}\right] \frac{2 m_{N}}{\rho_{N}}, \\
\langle\bar{q} i D i D q\rangle_{N} & =\left[\langle\bar{q} i D i D q\rangle_{\rho_{N}}-\langle\bar{q} i D i D q\rangle_{\mathrm{vac}}\right] \frac{2 m_{N}}{\rho_{N}}, \\
\left\langle q^{\dagger} i D q\right\rangle_{N} & =\left[\left\langle q^{\dagger} i D q\right\rangle_{\rho_{N}}-\left\langle q^{\dagger} i D q\right\rangle_{\mathrm{vac}}\right] \frac{2 m_{N}}{\rho_{N}},
\end{aligned}
$$

and

$$
\begin{aligned}
& \left\langle\frac{\alpha_{s}}{\pi} G_{\mu \nu}^{a} G^{a \mu \nu}\right\rangle_{N} \\
& \quad=\left[\left\langle\frac{\alpha_{s}}{\pi} G_{\mu \nu}^{a} G^{a \mu \nu}\right\rangle_{\rho_{N}}-\left\langle\frac{\alpha_{s}}{\pi} G_{\mu \nu}^{a} G^{a \mu \nu}\right\rangle_{\mathrm{vac}}\right] \frac{2 m_{N}}{\rho_{N}} .
\end{aligned}
$$

The condensate $\langle\bar{q} i D i D q\rangle_{\rho_{N}}$ appearing in Eq. (41) can be calculated in terms of light quark condensates using equations $[28,89]$

$$
\langle\bar{q} i D i D q\rangle_{\rho_{N}}+\frac{1}{8}\left\langle\bar{q} g_{s} \sigma G q\right\rangle_{\rho_{N}}=0.3 \mathrm{GeV}^{2} \rho_{N}
$$

and

$$
\left\langle\bar{q} g_{s} \sigma G q\right\rangle_{\rho_{N}}=\lambda^{2}\langle\bar{q} q\rangle_{\rho_{N}}+3.0 \mathrm{GeV}^{2} \rho_{N} .
$$

In this article, we have used the condensate value $\left\langle q^{\dagger} i D q\right\rangle_{N}=0.18 \mathrm{GeV}^{2} \rho_{N}$ from the linear density approximations results [89]. Now, in Eq. (32), to calculate the values of two unknowns $a$ and $b$, we need one more equation, which can be obtained by differentiation of Eq. (32) with $z=\frac{1}{M^{2}}$, i.e.,

$$
a \frac{d}{d z} C_{a}+b \frac{d}{d z} C_{b}=\frac{d}{d z} C_{f} .
$$

By solving Eqs. (32) and (46) simultaneously, we get the following mathematical formulas to find $a$ and $b$,

$$
\begin{array}{r}
a=\frac{C_{f}\left(-\frac{d}{d z}\right) C_{b}-C_{b}\left(-\frac{d}{d z}\right) C_{f}}{C_{a}\left(-\frac{d}{d z}\right) C_{b}-C_{b}\left(-\frac{d}{d z}\right) C_{a}}, \\
b=\frac{C_{f}\left(-\frac{d}{d z}\right) C_{a}-C_{a}\left(-\frac{d}{d z}\right) C_{f}}{C_{b}\left(-\frac{d}{d z}\right) C_{a}-C_{a}\left(-\frac{d}{d z}\right) C_{b}} .
\end{array}
$$

The obtained values of $a$ and $b$ are used to calculate the mass shift and decay constant of $D$ mesons given by Eqs. (28) and (29), respectively. 


\section{In-medium decay width of higher charmonium states using ${ }^{3} P_{0}$ model}

In the present work, one objective is to calculate the decay width of higher charmonium states $[\psi(3686), \psi(3770)$, $\left.\chi_{c 0}(3414), \chi_{c 2}(3556)\right]$ to pseudoscalar $D \bar{D}$ mesons. In order to calculate this observable, we rely on the ${ }^{3} P_{0}$ model $[30,90-$
92], which is a quark-antiquark pair creation model. In this model, a light quark pair is generated in the ${ }^{3} P_{0}$ state (vacuum), and one of the quarks (antiquarks) is combined with the heavy charm quark from the decaying charmonium at zero momentum. The matrix element for the decay $C \rightarrow D+\bar{D}$ (where $C$ is charmonia) is given as [30]

$$
M_{C \rightarrow D \bar{D}} \propto \int d^{3} k_{c} \phi_{C}\left(2 k_{c}-2 k_{D}\right) \phi_{D}\left(2 k_{c}-k_{D}\right) \phi_{\bar{D}}\left(2 k_{c}-k_{D}\right)\left[\bar{u}_{k_{c}, s} v_{-k_{c}, s}\right] .
$$

In the above, $k_{c}-k_{D}$ and $k_{D}-k_{c}$ represent the momentum of the charm quark and the charm antiquark of charmonia $C$. Since the decaying particle is assumed to be at rest, the magnitude of the momentum of $D$ and $\bar{D}$ meson is the same, i.e., $\left|k_{D}\right|=\left|k_{\bar{D}}\right|$. The term $\left[\bar{u}_{k_{c}, s} v_{-k_{c}, s}\right]$ denotes the wave function of the quark-antiquark pair in the vacuum and for the charmonium. We start with the harmonic oscillator wave function [24,30],

$$
\psi_{n L M_{L}}=(-1)^{n}(-\iota)^{L} R^{L+\frac{3}{2}} \sqrt{\frac{2 n !}{\Gamma\left(n+L+\frac{3}{2}\right)}} \exp \left(\frac{-R^{2} k^{2}}{2}\right) L_{n}^{L+\frac{1}{2}}\left(R^{2} k^{2}\right) Y_{l m}(\mathbf{k}),
$$

where $L_{n}^{L+\frac{1}{2}}\left(R^{2} k^{2}\right)$ denotes associate Laguerre polynomial, $Y_{l m}(\mathbf{k})$ represents the spherical harmonics, and $R$ is the radius of the charmonia.

Further, the decay rate of the charmonium state decaying into a $D \bar{D}$ pair can be represented as $[24,93]$

$$
\Gamma(C \rightarrow D+\bar{D})=2 \pi \frac{p_{D} E_{D} E_{\bar{D}}}{m_{C}}\left|M_{L S}\right|^{2} .
$$

Here $E_{D}=\sqrt{m_{D}^{2 *}+P_{D}^{2}}, E_{\bar{D}}=\sqrt{m_{\bar{D}}^{2 *}+P_{D}^{2}}$, and the center-of-mass momentum

$$
p_{D}=\left[\frac{m_{C}^{2}}{4}-\frac{m_{D}^{2 *}+m_{\bar{D}}^{2 *}}{2}+\frac{\left(m_{D}^{2 *}-m_{\bar{D}}^{2 *}\right)^{2}}{4 m_{C}^{2}}\right]^{1 / 2} .
$$

In the above, $m_{C}$ is the mass of charmonia and $M_{L S}$ is the invariant matrix amplitude.

Using Eq. (50), the decay rate of different higher charmonium states can be represented as $[28,30,93]$

$$
\begin{aligned}
\Gamma_{\psi(3686) \rightarrow D \bar{D}} & =\frac{\pi^{1 / 2} E_{D} E_{\bar{D}}}{m_{\psi}(3686)} \gamma^{2} \frac{2^{7}\left(3+2 r^{2}\right)^{2}\left(1-3 r^{2}\right)^{2}}{3^{2}\left(1+2 r^{2}\right)^{7}} y^{3}\left[1+\frac{2 r^{2}\left(1+r^{2}\right)}{\left(1+2 r^{2}\right)\left(3+2 r^{2}\right)\left(1-3 r^{2}\right)} y^{2}\right]^{2} e^{-\frac{y^{2}}{2\left(1+2 r^{2}\right)}}, \\
\Gamma_{\psi(3770) \rightarrow D \bar{D}} & =\frac{\pi^{1 / 2} E_{D} E_{\bar{D}}}{m_{\psi}(3770)} \gamma^{2} \frac{2^{11} 5}{3^{2}}\left(\frac{r}{1+2 r^{2}}\right)^{7} y^{3}\left[1-\frac{1+r^{2}}{5\left(1+2 r^{2}\right)} y^{2}\right]^{2} e^{-\frac{y^{2}}{2\left(1+2 r^{2}\right)}} \\
\Gamma_{\chi_{c 0}(3414) \rightarrow D \bar{D}} & =\pi^{1 / 2} \frac{E_{D} E_{\bar{D}}}{2 m_{\chi_{c 0}}(3414)} \gamma^{2} 2^{9} 3\left(\frac{r}{1+2 r^{2}}\right)^{5} y\left[1-\frac{1+r^{2}}{3\left(1+2 r^{2}\right)} y^{2}\right]^{2} e^{-\frac{y^{2}}{2\left(1+2 r^{2}\right)}}
\end{aligned}
$$

and

$$
\Gamma_{\chi_{c 2}(3556) \rightarrow D \bar{D}}=\frac{\pi^{1 / 2} E_{D} E_{\bar{D}}}{m_{\chi_{c 2}}(3556)} \gamma^{2} \frac{2^{10} r^{5}\left(1+r^{2}\right)^{2}}{15\left(1+2 r^{2}\right)^{7}} y^{5} e^{-\frac{y^{2}}{2\left(1+2 r^{2}\right)}} .
$$

In the above equations, the variables $r$ and $\beta_{D}$ incorporate the modification of the wave function due to the nodal structure of the initial- and final-state mesons [94,95] and their values are fitted with the help of the experimental partial decay width of $\psi(4040)$ to $D \bar{D}$ mesons [30]. The parameter $y$ is expressed as, $y=\frac{p_{D}}{\beta_{D}}$ and the parameter $\gamma$ denotes the strength of the vertex and fitted using the experimental decay width of $\Gamma[\psi(3770) \rightarrow D \bar{D}][28,30]$. The decay width of different higher charmonia can be calculated by the above equations by using the effective mass of $D$ meson obtained from the QCDSR calculations.

\section{NUMERICAL RESULTS AND DISCUSSIONS}

In this section, we will discuss our observations on the effect of the magnetic field on masses and decay constants of pseudoscalar $\left(D^{+}, D^{0}\right)$ and scalar $\left(D_{0}^{+}, D_{0}^{0}\right)$ mesons and in-medium decay widths of higher charmonium states $\left[\psi(3686), \psi(3770), \chi_{c 0}(3414)\right.$ and $\left.\chi_{c 2}(3556)\right]$ in asymmetric nuclear matter at finite temperature. As discussed earlier, the light quark condensates and gluon condensates have been calculated by using the chiral SU(3) model and the different parameters used in the model are shown in Table I. In addition, the value of charm quark mass $m_{c}$, running coupling constant $\alpha_{s}$, coupling constant $g_{\mathrm{DNH}}$, and constant $\lambda$ are approximated to be $1.3,0.45,6.74$, and $0.5 \mathrm{GeV}$, respectively $[28,63]$. The vacuum masses of $D$ mesons are taken as $1.869,1.864,2.355$, and $2.350 \mathrm{GeV}$ for $D^{+}, D^{0}, D_{0}^{+}$, and $D_{0}^{0}$ mesons, respectively. The vacuum values of the decay constant for pseudoscalar and 
TABLE I. Values of different parameters.

\begin{tabular}{lcccc}
\hline \hline$k_{0}$ & $k_{1}$ & $k_{2}$ & $k_{3}$ & $k_{4}$ \\
\hline 2.53 & 1.35 & -4.77 & -2.77 & -0.218 \\
$\sigma_{0}(\mathrm{MeV})$ & $\zeta_{0}(\mathrm{MeV})$ & $\chi_{0}(\mathrm{MeV})$ & $d$ & $\rho_{0}\left(\mathrm{fm}^{-3}\right)$ \\
-93.29 & -106.8 & 409.8 & 0.064 & 0.15 \\
$m_{\pi}(\mathrm{MeV})$ & $m_{K}(\mathrm{MeV})$ & $f_{\pi}(\mathrm{MeV})$ & $f_{K}(\mathrm{MeV})$ & $g_{4}$ \\
139 & 498 & 93.29 & 122.14 & 79.91 \\
$g_{\sigma N}$ & $g_{\zeta N}$ & $g_{\delta N}$ & $g_{\omega N}$ & $g_{\rho N}$ \\
10.56 & -0.46 & 2.48 & 13.35 & 5.48 \\
\hline \hline
\end{tabular}

scalar mesons are taken as 0.210 and $0.334 \mathrm{GeV}$, respectively [24,28]. Furthermore, the continuum threshold parameter $s_{0}$ for pseudoscalar and scalar mesons are taken as 6.2 and 8 $\mathrm{GeV}^{2}$, respectively [63]. We have chosen the proper Borel window so that there will be a least variation in the mass shift $\left(\Delta m_{D}^{*}\right)$ and decay shift $\left(\Delta f_{D}^{*}\right)$. The Borel window for masses of $\left(D^{+}, D^{0}\right)$ and $\left(D_{0}^{+}, D_{0}^{0}\right)$ are taken as $4.5-5.5$ and 6-7 $\mathrm{GeV}^{2}$, respectively, whereas the range of Borel window for the decay constants of $\left(D^{+}, D^{0}\right)$ and $\left(D_{0}^{+}, D_{0}^{0}\right)$ are taken as 2-3 and 7-9 $\mathrm{GeV}^{2}$, respectively. Moreover, the values of parameters $\gamma$, $\beta_{D}$, and $r$ used in the ${ }^{3} P_{0}$ model are taken as $0.281,0.30$, and 1.04 , respectively [28]. Our further discussion of this section is divided into three subsections.

\section{A. Magnetic-field-induced condensates and mass of nucleons}

In this subsection, we show the results for the mediuminduced light quark and gluon condensates, which are calculated using the chiral model described in Sec. II A. From the expressions given in Eqs. (15)-(17), one can see that the condensates depend on the scalar fields $\sigma, \zeta, \delta$, and $\chi$, which are solved under different conditions of medium such as density, magnetic field, temperature, and asymmetry [9,74]. In Figs. 1 and 2, we have plotted the scalar up-quark condensate $\langle\bar{u} u\rangle_{\rho_{N}}$, down-quark condensate $\langle\bar{d} d\rangle_{\rho_{N}}$, and gluon condensate $\left\langle\frac{\alpha_{s}}{\pi} G_{\mu \nu}^{a} G^{a \mu \nu}\right\rangle_{\rho_{N}}$ with respect to magnetic field at isospin symmetry parameters $\eta=0$ and 0.5 , respectively. We have shown the results at nucleon densities $\rho_{N}=\rho_{0}$ and $4 \rho_{0}$ and temperatures $T=0,50,100$, and $150 \mathrm{MeV}$. In Fig. 1, at $\eta=0$, we can see that the magnitude of the up- and downquark condensates decreases with the increase in the magnetic field. One can also conclude that the density effects are also appreciable as the magnitude of quark condensates decrease with the increase in the density. Moreover, inclusion of temperature effects increase the magnitude of quark condensates but the trend concerning the magnetic field remains the same. For example, in symmetric nuclear medium for $e B=4 m_{\pi}^{2}$, the values of $\langle\bar{u} u\rangle_{\rho_{N}}\left(\langle\bar{d} d\rangle_{\rho_{N}}\right)$, at $\rho_{N}=\rho_{0}$, is $-10.28(-7.42)$, $-11.16(-8.02)$, and $-11.41(-8.18)$ times $10^{-3} \mathrm{GeV}^{3}$ for $T=0,100$, and $150 \mathrm{MeV}$, respectively, and for $\rho_{N}=4 \rho_{0}$ it modifies to $-4.81(-3.61),-5.40(-4.10)$, and -5.79 $(-4.27)$ times $10^{-3} \mathrm{GeV}^{3}$. It may be noted that despite $\eta=0$, the value of up- and down-quark condensates are different, which is contradictory to the previous work (at zero magnetic field) as up- and down-quark are isospin partners and hence indistinguishable in symmetric nuclear matter [78]. This is because of Landau quantization, which occurs due to the
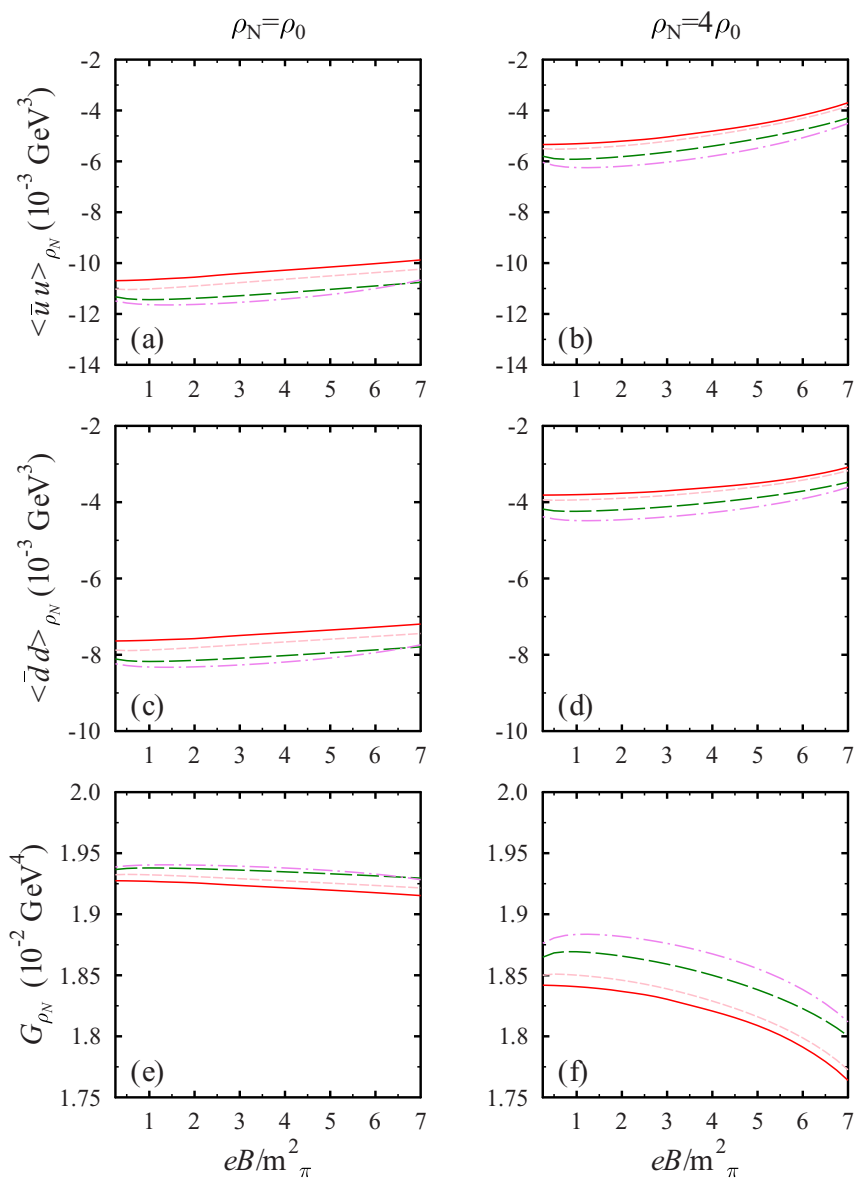

\begin{tabular}{|l|}
\hline$T=0 \mathrm{MeV}--T=100 \mathrm{MeV}$ \\
$-T=50 \mathrm{MeV}-T=150 \mathrm{MeV}$
\end{tabular}

FIG. 1. The light quark condensates $\left(\langle\bar{u} u\rangle_{\rho_{N}}\right.$ and $\left.\langle\bar{d} d\rangle_{\rho_{N}}\right)$ and gluon condensate $\left\langle\frac{\alpha_{s}}{\pi} G_{\mu \nu}^{a} G^{a \mu \nu}\right\rangle_{\rho_{N}}$ (denoted by $G_{\rho_{N}}$ ) is plotted for symmetric nuclear matter $(\eta=0)$ as a function of magnetic field $e B$ under different conditions of medium.

interaction of charged protons with the magnetic field. This interaction disturbs the equality between the scalar density of protons and neutrons and hence the magnitude of $\delta$ field become nonzero [74].

In Figs. 1(e) and 1(f), we also show the results for scalar gluon condensate and observed that the magnitude of the gluon condensate changes much less as compared to light quark condensates. This is why open charm mesons experience a larger mass shift than the ground-state charmonia $[9,28]$. The value of gluon condensate at nuclear saturation density decreases as a function of the magnetic field and this trend becomes more appreciable at $\rho_{N}=4 \rho_{0}$. Furthermore, we observe that the temperature effects on gluon condensate are opposite those of quark condensates. This is because the gluon condensate depends on the fourth power of the $\chi$ field along with the $\sigma$ and $\zeta$ fields [see Eq. (17), whereas quark condensates [see Eq. (15) and (16)] hae only $\sigma$ - and $\delta$-field dependence [74].

In Fig. 2, at $\eta=0.5$, we observe similar behavior of condensates as a function of the magnetic field as was at $\eta=0$, except at low temperature. For example, at $e B=4 m_{\pi}^{2}$, 

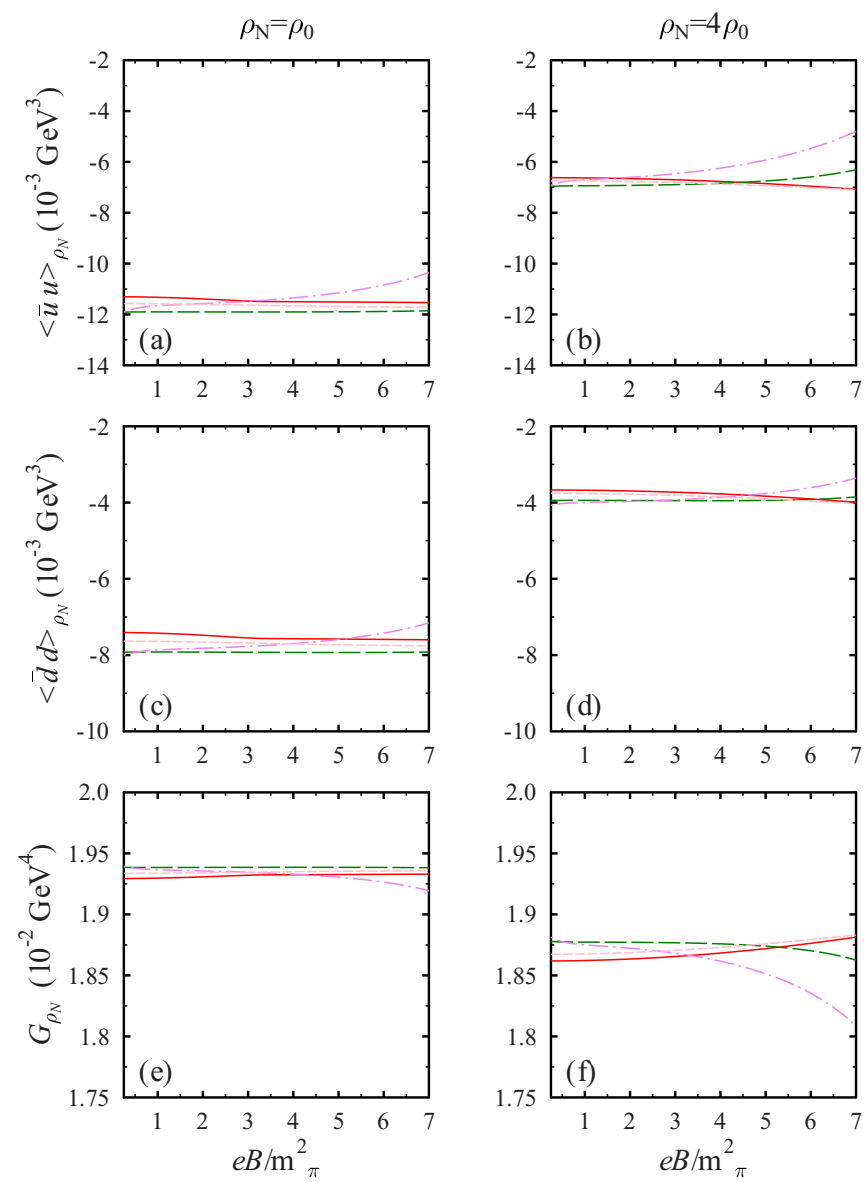

\begin{tabular}{|l|}
\hline$T=0 \mathrm{MeV}--T=100 \mathrm{MeV}$ \\
$--T=50 \mathrm{MeV}-T=150 \mathrm{MeV}$ \\
\hline
\end{tabular}

FIG. 2. The light quark condensates $\left(\langle\bar{u} u\rangle_{\rho_{N}}\right.$ and $\left.\langle\bar{d} d\rangle_{\rho_{N}}\right)$ and gluon condensate $\left\langle\frac{\alpha_{s}}{\pi} G_{\mu \nu}^{a} G^{a \mu \nu}\right\rangle_{\rho_{N}}$ (denoted by $G_{\rho_{N}}$ ) is plotted for asymmetric nuclear matter $(\eta \neq 0)$ as a function of magnetic field $e B$ under different conditions of medium.

the values of $\langle\bar{u} u\rangle_{\rho_{N}}\left(\langle\bar{d} d\rangle_{\rho_{N}}\right)$ at $\rho_{N}=\rho_{0}$ are $-11.49(-7.57)$, $-11.90(-7.93)$, and $-11.34(-7.69)$ times $10^{-3} \mathrm{GeV}^{3}$ for $T=0,100$, and $150 \mathrm{MeV}$, respectively, and for $\rho_{N}=4 \rho_{0}$ it modifies to $-6.76(-3.77),-6.84(-3.95)$, and -6.24 $(-3.86)$ times $10^{-3} \mathrm{GeV}^{3}$. We also observed a crossover behavior in the plot of gluon condensate. In this case, the value of scalar gluon condensate increases for low temperature, whereas it decreases for high temperature. This is because in a medium having a large number of neutrons, the behavior of the neutron scalar density in low-temperature modifies, hence the $\delta$ field also modifies [15,74]. Therefore, from Eqs. (15) and (16), one can see that in symmetric nuclear matter, these quark condensates are directly proportional to scalar fields $\sigma$ and $\delta$, and hence the behavior of $\langle\bar{q} q\rangle_{\rho_{N}}$ with different medium parameters is same as that of the $\sigma$ field (as the $\delta$ field has much less variation with the magnetic field for $\eta=0$ ) [74]. On the other hand, for asymmetric matter, the condensates have mixed contributions of $\sigma$ and $\delta$ fields as the $\delta$ field varies appreciably with the increase in the magnetic field. Moreover, the gluon condensate has the dependence on scalar fields $\sigma$, $\zeta$, and $\chi$. The scalar fields as a function of magnetic field

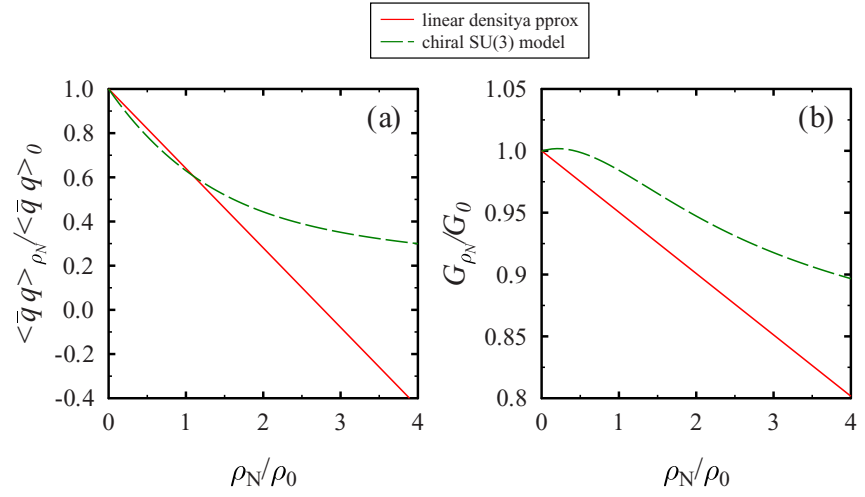

FIG. 3. Comparison of quark $\langle\bar{q} q\rangle_{\rho_{N}}$ and gluon condensates $\left\langle\frac{\alpha_{s}}{\pi} G_{\mu \nu}^{a} G^{a \mu \nu}\right\rangle_{\rho_{N}}$ (denoted by $G_{\rho_{N}}$ ) calculated in the chiral SU(3) model and in the linear density approximation.

with different value of density, asymmetry, and temperature are plotted and discussed in our previous work [74]. As per inverse magnetic catalysis, the scalar fields gets enhanced due to the generation of additional fermion antifermion condensates in the presence of magnetic field $[6,69,74]$.

Furthermore, in order to understand the validity of our calculations of quark and gluon condensates at higher density of nuclear matter, in Fig. 3 we compare the quark and gluon condensate using the chiral SU(3) model with that of linear density approximation. In literature, the quark condensate in linear density approximation has been calculated by the expression $\langle\bar{q} q\rangle_{\rho_{N}}=\langle\bar{q} q\rangle_{0}+\frac{\sigma_{N} \rho_{N}}{m u+m d}$, where $\sigma_{N}$ is taken as $45 \mathrm{MeV}$ [63] and $\langle\bar{q} q\rangle_{0}$ is the vacuum value of quark condensate. In addition, in the same approximation gluon condensates have been calculated by the expression $G_{\rho_{N}}=G_{0}-$ $\frac{8}{9} m_{N} \rho_{N}$ [96], where $G_{0}$ is vacuum value of gluon condensate and $m_{N}$ is vacuum value of nucleon mass. On the other side, the quark and gluon condensate are evaluated from the chiral model using Eqs. (15)-(17). From Fig. 3, we observe that in linear density approximation the quark and gluon condensate decreases linearly with density, whereas in chiral-model calculations, the condensates shows slow decrease (nonlinear) with respect to density. A similar response of quark and gluon condensate in the medium has been observed in Refs. [96-99]. Therefore, the quark and gluon condensates evaluated in the chiral model validate us to calculate observables in high density and these calculations are not exactly in linear density approximation.

In Fig. 4, we have shown the magnetic-field-induced mass $\left(m_{i}^{*}\right)$ of proton and neutron under the effect of different values of temperature, magnetic field, density, and isospin asymmetry. In this figure, for symmetric matter $(\eta=0)$, we observe the mass of proton/neutron decrease with the increase in magnetic field. We also observed the mass of nucleons decreases heavily with the increase in density and follows the same trend as a function of magnetic field. Inclusion of temperature effects shows increase in mass at particular value of magnetic field. This is because in chiral model, the mass of nucleons are calculated on the basis of scalar fields $\sigma, \zeta$, and $\delta$ by the relation $m_{i}^{*}=-\left(g_{\sigma i} \sigma+g_{\zeta i} \zeta+g_{\delta i} \tau_{3 i} \delta\right)$, which are solved under the influence of scalar density of nucleons. 

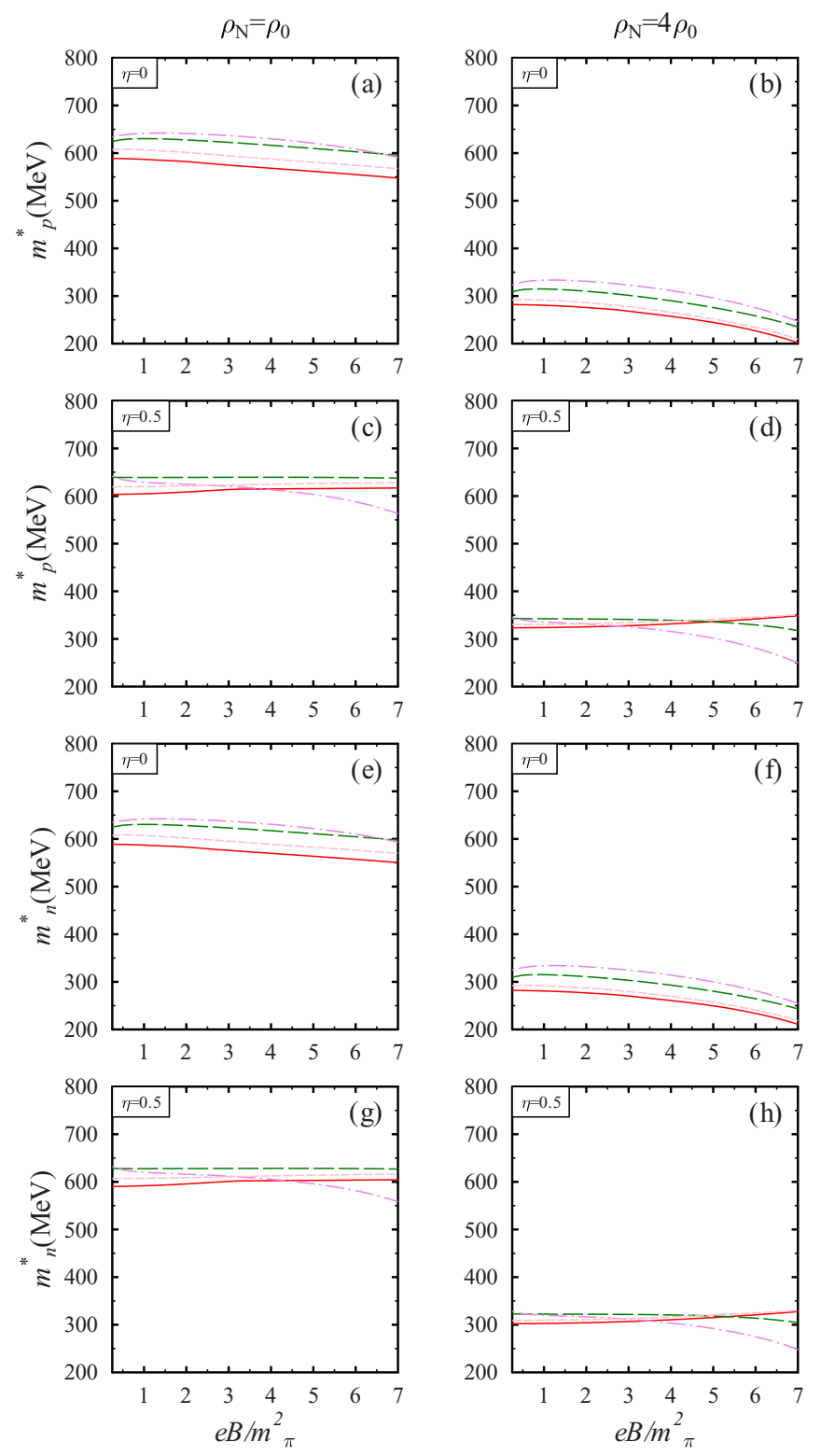

$\begin{array}{r}-T=0 \mathrm{MeV}--T=100 \mathrm{MeV} \\ T=50 \mathrm{MeV}-T=150 \mathrm{MeV} \\ \hline\end{array}$

FIG. 4. The effective masses of protons and neutrons are plotted for symmetric and asymmetric nuclear matter as a function of magnetic field $e B$ under different conditions of medium.

One can see in spite of symmetric nuclear matter, the masses of protons and neutrons are slightly different. For example, at $e B=4 m_{\pi}^{2}$ and $\eta=0$, the values of $m_{p}^{*}\left(m_{n}^{*}\right)$ at $\rho_{N}=\rho_{0}$ is 630.03 (630.76), 616.44 (617.35), and 568.24 (569.78) MeV for $T=0,100$, and $150 \mathrm{MeV}$, respectively, and for $\rho_{N}=4 \rho_{0}$ it modifies to 257.3 (260.80), 289.98 (293.06), and 311.45 (314.07) MeV. As discussed earlier, this is because of nonzero value of $\delta$ field, which arises due to the Landau quantization. On the other hand, for asymmetric nuclear matter, the mass of nucleons decreases as a function of density (similar in symmetric matter). Furthermore, the mass of nucleon decreases for large values of temperature but increases for lower values of temperature as a function of magnetic field. Here the mass
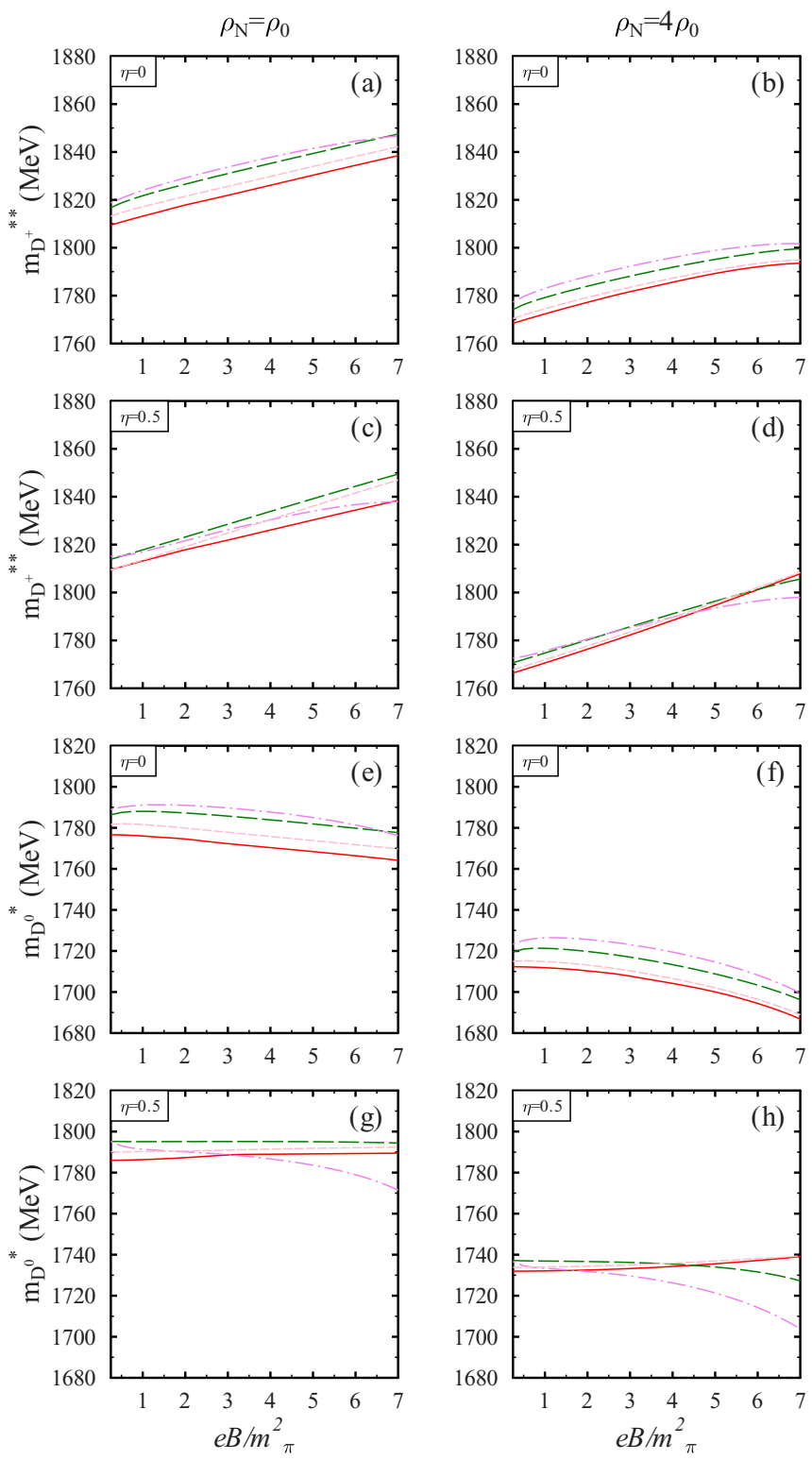

FIG. 5. The effective mass of pseudoscalar $D^{+}$(charged) and $D^{0}$ (uncharged) mesons is plotted as a function of magnetic field $e B$ under different conditions of medium.

splitting is larger as in case of symmetric matter. For example, at $e B=4 m_{\pi}^{2}$ and $\eta=0.5$, the values of $m_{p}^{*}\left(m_{n}^{*}\right)$ at $\rho_{N}=\rho_{0}$ is 614.96 (602.46), 669.44 (628.35), and 614.96 (602.46) MeV for $T=0,100$, and $150 \mathrm{MeV}$, respectively, and for $\rho_{N}=$ $4 \rho_{0}$ it modifies to 331.52 (310.45), 339.12 (320.58), and 315.63 (303.74) MeV. In asymmetric matter the $\delta$ field varies appreciably and due to Landau effects the scalar densities of proton and neutron generate this crossover behavior.

\section{B. Mass and shift in decay constant of pseudoscalar and scalar $D$ mesons}

Here we will discuss how mass and shift in decay constants of isospin-averaged pseudoscalar $\left(D^{+}, D^{0}\right)$ and scalar 
TABLE II. The values of the magnetic-field-induced masses of $D^{+}, D^{0}, D_{0}^{0}$, and $D_{0}^{+}$mesons (in units of MeV).

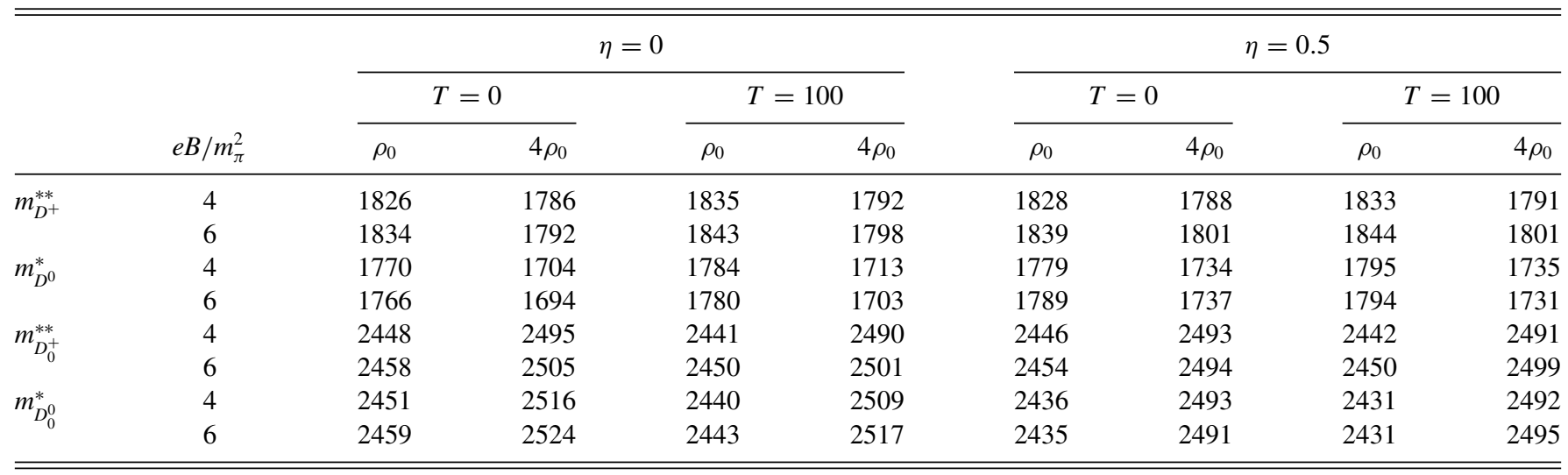

$\left(D_{0}^{+}, D_{0}^{0}\right)$ mesons modifies with magnetic field and other medium properties. As discussed earlier in Sec. II B, the above observables are calculated in the QCDSR by using quark and gluon condensates. In Figs. 5 and 7, we have plotted the masses of pseudoscalar and scalar $D$ mesons, respectively, as a function of external magnetic field in nuclear matter. The values of the effective mass of pseudoscalar and scalar $D$ mesons in the presence of magnetic field and other medium properties are shown in Table II for better comparison. In Fig. 5, for symmetric nuclear matter, we observe that the effective mass of charged $D^{+}$meson increases with the increase in magnetic field and hence the magnitude of negative mass shift of $D^{+}$meson decreases. For example, at $\eta=0$, $\rho_{N}=\rho_{0}$, and $T=0$, the value of mass increase from 1826 to $1834 \mathrm{MeV}$, when we move from $e B=4 m_{\pi}^{2}$ to $6 m_{\pi}^{2}$. This is because the $D^{+}$meson is a charged meson and with the interaction of a magnetic field additional positive mass shift [see Eq. (31)] comes into the picture. If we do not consider the additional mass shift, then we observe a negative in-medium mass shift which increases with the increase in magnetic field. The density effect on the mass shift of $D^{+}$meson is also very prominent as the mass shift of $D^{+}$meson increase with the increase in density. For instance, at $e B=4 m_{\pi}^{2}, \eta=0$, and $T=0 \mathrm{MeV}$, when we move from $\rho_{N}=\rho_{0}$ to $4 \rho_{0}$, the values of mass change from 1826 to $1786 \mathrm{MeV}$. The inclusion of temperature effects increases the mass at particular values of density. For example, at $\eta=0, e B=4 m_{\pi}^{2}$, and $\rho_{N}=4 \rho_{0}$, when we move from $T=0$ to $100 \mathrm{MeV}$, the values of mass change from 1786 to $1792 \mathrm{MeV}$.

For neutral pseudoscalar $D^{0}$ meson there will not be any additional positive mass shift [see Eq. (31)] and therefore the effective mass in this case does not increase but decreases with the increase in magnetic field (see Table II). However, the temperature and density effects remain the same as for $D^{+}$meson. For instance, at $e B=4 m_{\pi}^{2}, \eta=0$, and $T=0$ (100) $\mathrm{MeV}$, the mass of $D^{0}$ meson is 1770 (1784) and 1704 (1713) $\mathrm{MeV}$ for $\rho_{N}=\rho_{0}$ and $4 \rho_{0}$, respectively, and for $e B=$ $6 m_{\pi}^{2}$, the value changes to 1766 (1780) and 1694 (1703) MeV. It is observed that for both pseudoscalar mesons, if we go from symmetric matter to highly asymmetric matter, then the effect of temperature become less appreciable except for $T=150 \mathrm{MeV}$. The isospin asymmetry effects should be quite visible for $D^{+}(c \bar{d})$ and $D^{0}(c \bar{u})$ mesons as they are isospin partner of each other. For nonmagnetic nuclear matter, appreciable isospin effects are observed [28]. In Ref. [28], the mass of the $D^{0}$ meson decreased, whereas the mass of $D^{+}$increases as we go from symmetric to asymmetric nuclear matter. But in the present case, the asymmetry effects on the $D^{+}$meson are

TABLE III. Magnetic-field-induced mass shift of $D^{+}$and $D^{0}$ mesons (in MeV) at $e B=4 m_{\pi}^{2}$ are compared with the mass shift obtained without magnetic field [28]. We have also considered and compared the contribution of other quark condensates (OQC).

\begin{tabular}{|c|c|c|c|c|c|c|c|c|c|c|}
\hline & & & \multicolumn{4}{|c|}{$\eta=0$} & \multicolumn{4}{|c|}{$\eta=0.5$} \\
\hline & All condensates & $\Delta m^{* *}$ & -43 & -83 & -34 & -77 & -41 & -81 & -36 & -78 \\
\hline \multirow[t]{3}{*}{$D^{+}$} & & $\Delta m(B=0)$ & -64 & -110 & -55 & -103 & -68 & -112 & -60 & -108 \\
\hline & $\mathrm{OQC}=0$ & $\Delta m^{* *}$ & -60 & -92 & -51 & -85 & 58 & -89 & -52 & -86 \\
\hline & All condensates & $\Delta m^{*}$ & -94 & -160 & -80 & -151 & -85 & -130 & -69 & -129 \\
\hline \multirow[t]{3}{*}{$D^{0}$} & & $\Delta m(B=0)$ & -92 & -163 & -79 & -153 & -81 & -141 & -72 & -137 \\
\hline & $\mathrm{OQC}=0$ & $\Delta m^{*}$ & -89 & -146 & -76 & -137 & -71 & -116 & -65 & -115 \\
\hline & & $\Delta m(B=0)$ & -90 & -154 & -77 & -144 & -79 & -133 & -69 & -128 \\
\hline
\end{tabular}


TABLE IV. Magnetic-field-induced mass shift of $D_{0}^{+}$and $D_{0}^{0}$ mesons (in MeV) at $e B=4 m_{\pi}^{2}$ are compared with the mass shift obtained without magnetic field [24]. We have also considered and compared the contribution of additional condensates.

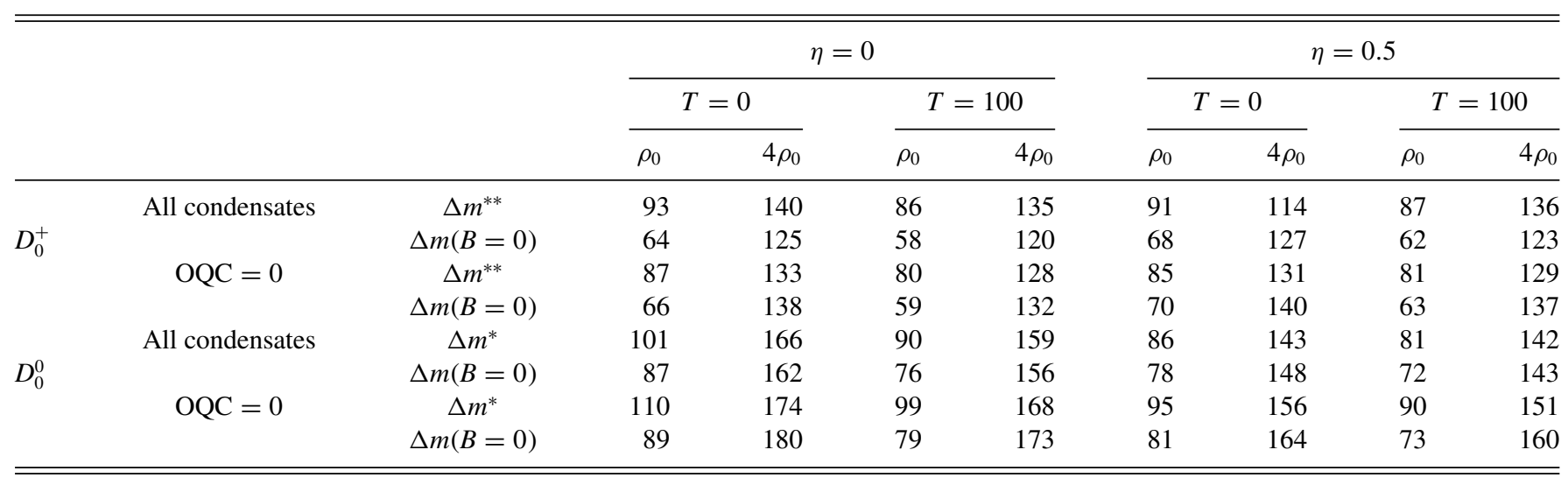

compensated for by the additional positive mass shift due to Landau interaction, and hence we see fewer crossover temperature effects. On the other hand, the effective mass of the $D^{0}$ meson modifies appreciably in asymmetric nuclear matter and shows crossover behavior. It shows a slight increase for high temperature, but for low temperature it decreases appreciably. For example, at $e B=6 m_{\pi}^{2}, \eta=0.5$, and $T=0$ (100) $\mathrm{MeV}$, the mass of the $D^{0}$ meson is 1789 (1794) and 1737 (1731) $\mathrm{MeV}$ for $\rho_{N}=\rho_{0}$ and $4 \rho_{0}$, respectively. This crossover is a reflection of the behavior of quark and gluon condensates in asymmetric magnetized nuclear matter as shown in Fig. 2.

As can be seen from Fig. 7, contrary to pseudoscalar $D$ mesons, we observed a positive mass shift for scalar $D_{0}^{+}$and $D_{0}^{0}$ mesons. The fact that the effective mass will decrease or increase depends on the sign of the scattering length [see Eq. (27)]. In heavy meson-nucleon bound states, the negative or positive sign of scattering length determines whether the $D N$ interactions are attractive or repulsive [63]. The enhancement in mass of the $D_{0}^{+}$meson is more than the $D_{0}^{0}$ meson as a function of magnetic field. As discussed, the charged meson experiences additional positive shift due to induced Landau levels. For scalar $D$ mesons, we observed an opposite behavior of masses as a function of temperature comparative to the pseudoscalar case. In symmetric nuclear matter, the effective mass of the scalar $D$ meson decreases with the increase in temperature while follows the same trend as a function of magnetic field. The asymmetric effects on scalar $D_{0}^{+}$meson are also compensated for by the magnetic field as for the pseudoscalar case.

As we pointed out, condensates $\left\langle q^{\dagger} i D q\right\rangle_{N}$ and $\langle\bar{q} i D i D q\rangle_{N}$ have less effect as compared to $\langle\bar{q} q\rangle_{N}$ condensates on inmedium properties of charmed mesons (see Tables III and IV for pseudoscalar and scalar mesons, respectively). In these tables, we have also compared the mass shift with and without magnetic effect. The results of mass shift in the absence of magnetic field have been taken from Ref. [28] (pseudoscalar) and Ref. [24] (scalar). In this comparison, we observed that the presence of magnetic field affects the mass shift of charged pseudoscalar and scalar $D$ meson significantly, whereas for neutral $D$ meson the effects are good but not prominent as compared to charged meson. This is due to additional positive mass shift in the medium which occurs via Landau quantization as discussed earlier also.

In our knowledge, the in-medium masses of $D$ mesons at finite temperature and density of nuclear matter considering external magnetic fields have not been evaluated yet within any model. In Ref. [15] the mass of pseudoscalar $D$ meson in strongly magnetized asymmetric cold nuclear matter has been calculated solely in the effective chiral SU(4) model. In this article, the mass splitting between $D^{+}\left(D^{0}\right)$ and $D^{-}\left(\bar{D}^{0}\right)$ has

TABLE V. The values of magnetic-field-induced shift in decay constants of $D^{+}, D^{0}, D_{0}^{0}$, and $D_{0}^{+}$mesons (in units of MeV).

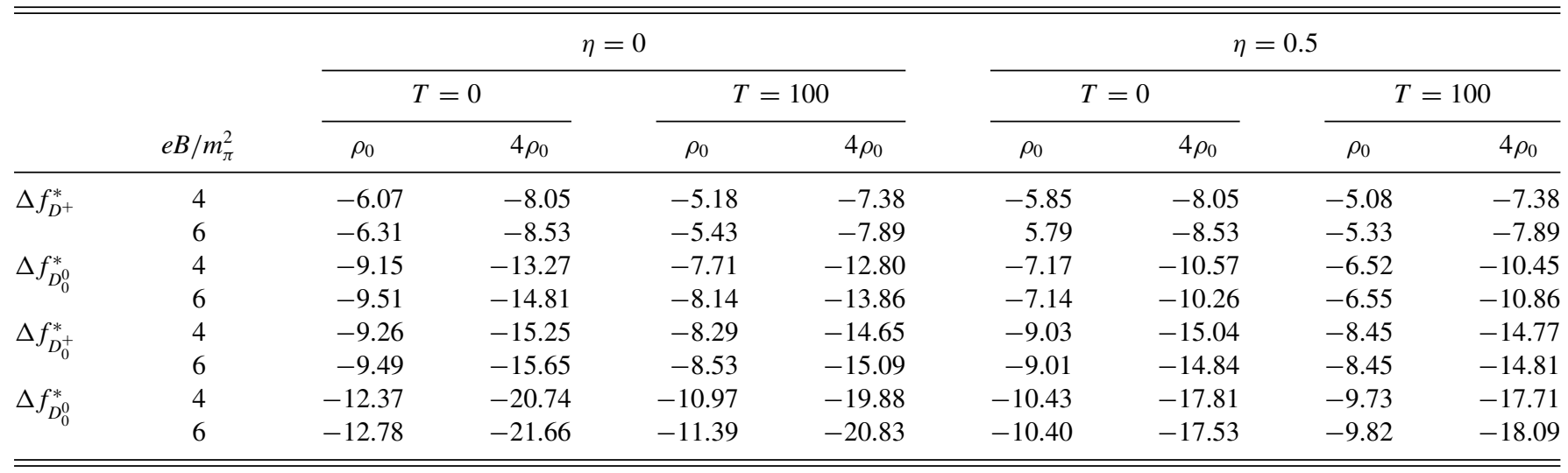



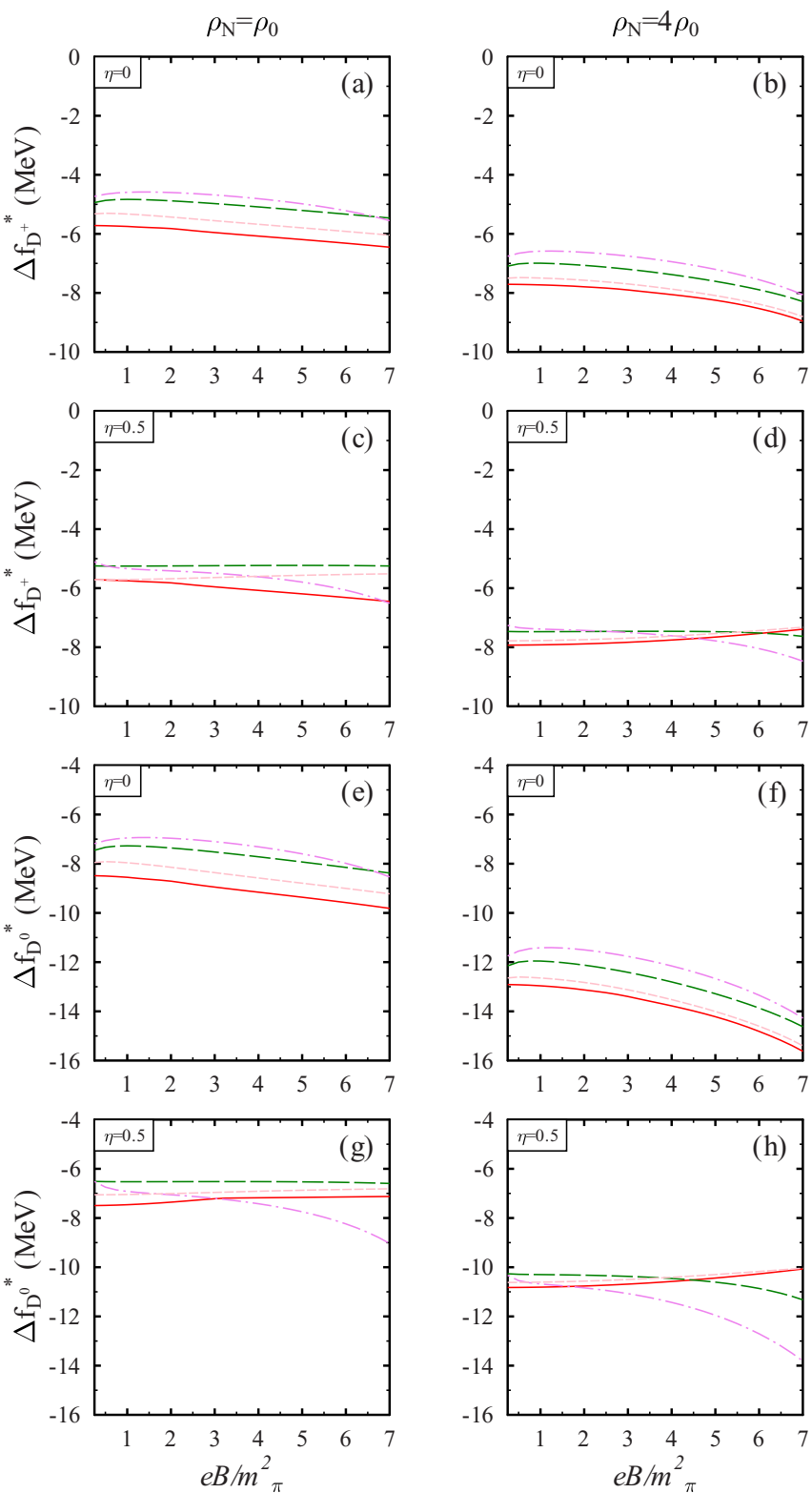

$T=0 \mathrm{MeV}-T=100 \mathrm{MeV}$
$T=50 \mathrm{MeV}-T=150 \mathrm{MeV}$

FIG. 6. The shift in decay constant of pseudoscalar $D^{+}$(charged) and $D^{0}$ (uncharged) mesons is plotted as a function of magnetic field $e B$ under different conditions of medium.

been calculated using the self-energy of $D$ mesons. In cold and asymmetric nuclear matter, the mass shift of $D$ mesons has been compared with and without taking contributions from anomalous magnetic moment (AMM). In Ref. [66], authors used hadronic QCD sum rules in which the magnetic-field effects are introduced on both phenomenological as well as the OPE side to evaluate the effect of magnetic field on $D$ meson properties (at zero density and temperature). The additional mixing effects are examined on the phenomenological side by adding a spectral ansatz term. Besides the mixing effects, an additional perturbative positive mass shift is also found due to
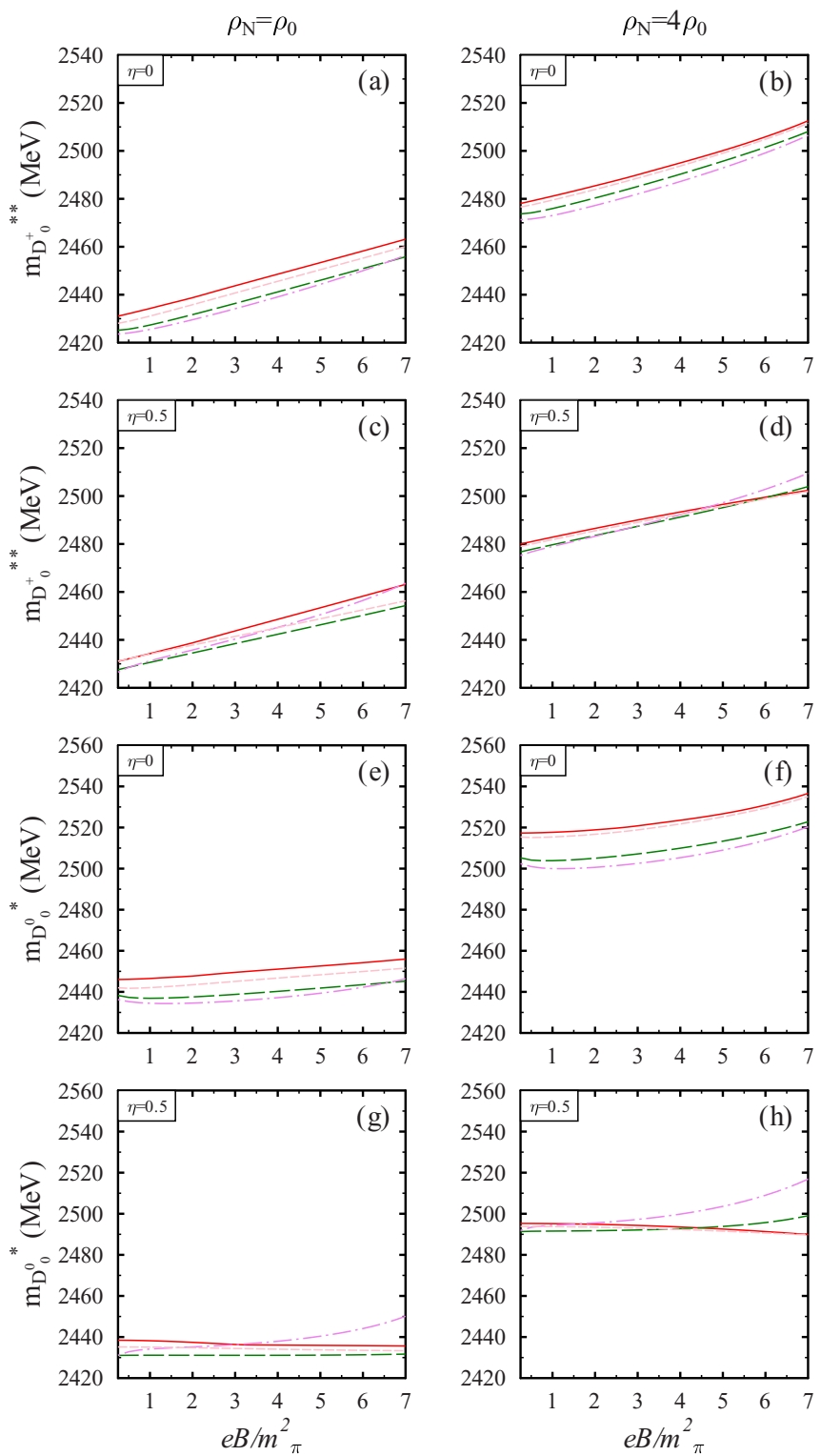

FIG. 7. The effective mass of scalar $D_{0}^{+}$(charged) and $D_{0}^{0}$ (uncharged) mesons is plotted as a function of magnetic field $e B$ under different conditions of medium.

the magnetic field. In our future work, we will also include the mixing effects of $D$ mesons in the presence of magnetic field.

In Fig. 6 (Fig. 8), for a given value of density, temperature, and isospin asymmetry the shift in decay constant $\Delta f_{D}^{*}$ of pseudoscalar (scalar) $D$ meson is plotted as a function of magnetic field. The values of in-medium shift in decay constant of pseudoscalar and scalar $D$ meson in the presence of magnetic field and other medium properties are shown in Table V. In symmetric nuclear matter, the magnitude of $\Delta f_{D}^{*}$ increases as a function of magnetic field, whereas it decreases with the increase in temperature. But for $\eta=0.5$, the crossover behavior is observed as was the case for effective masses. This is because the shift in decay constant is calculated using the 

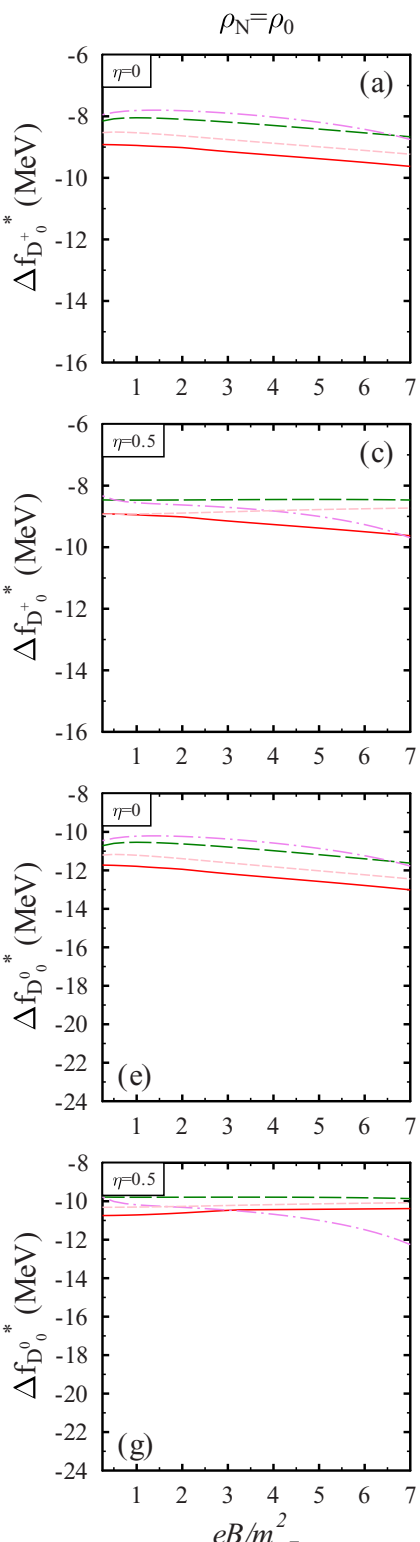

$e B / m^{2}$

$$
\begin{array}{r}
-T=0 \mathrm{MeV}--T=100 \mathrm{MeV} \\
-T=50 \mathrm{MeV}-T=150 \mathrm{MeV}
\end{array}
$$

FIG. 8. The shift in decay constant of scalar $D_{0}^{+}$(charged) and $D_{0}^{0}$ (uncharged) mesons is plotted as a function of magnetic field $e B$ under different conditions of medium.

shift in effective mass [see Eq. (29)] in the QCDSR. It may be noted that for scalar mesons, the shift in decay constant is negative despite the positive mass shift.

\section{In-medium decay width of higher charmonium states}

Now we will see how the obtained magnetically induced masses of pseudoscalar $D$ meson affect the in-medium decay width of higher charmonia $\left[\psi(3686), \psi(3770), \chi_{c 0}(3414)\right.$, $\left.\chi_{c 2}(3556)\right]$ decaying to $D \bar{D}$ pairs. We have neglected the medium modifications of the parent charmonia in the present work. In Figs. 9 and 10, we have shown the variation of partial
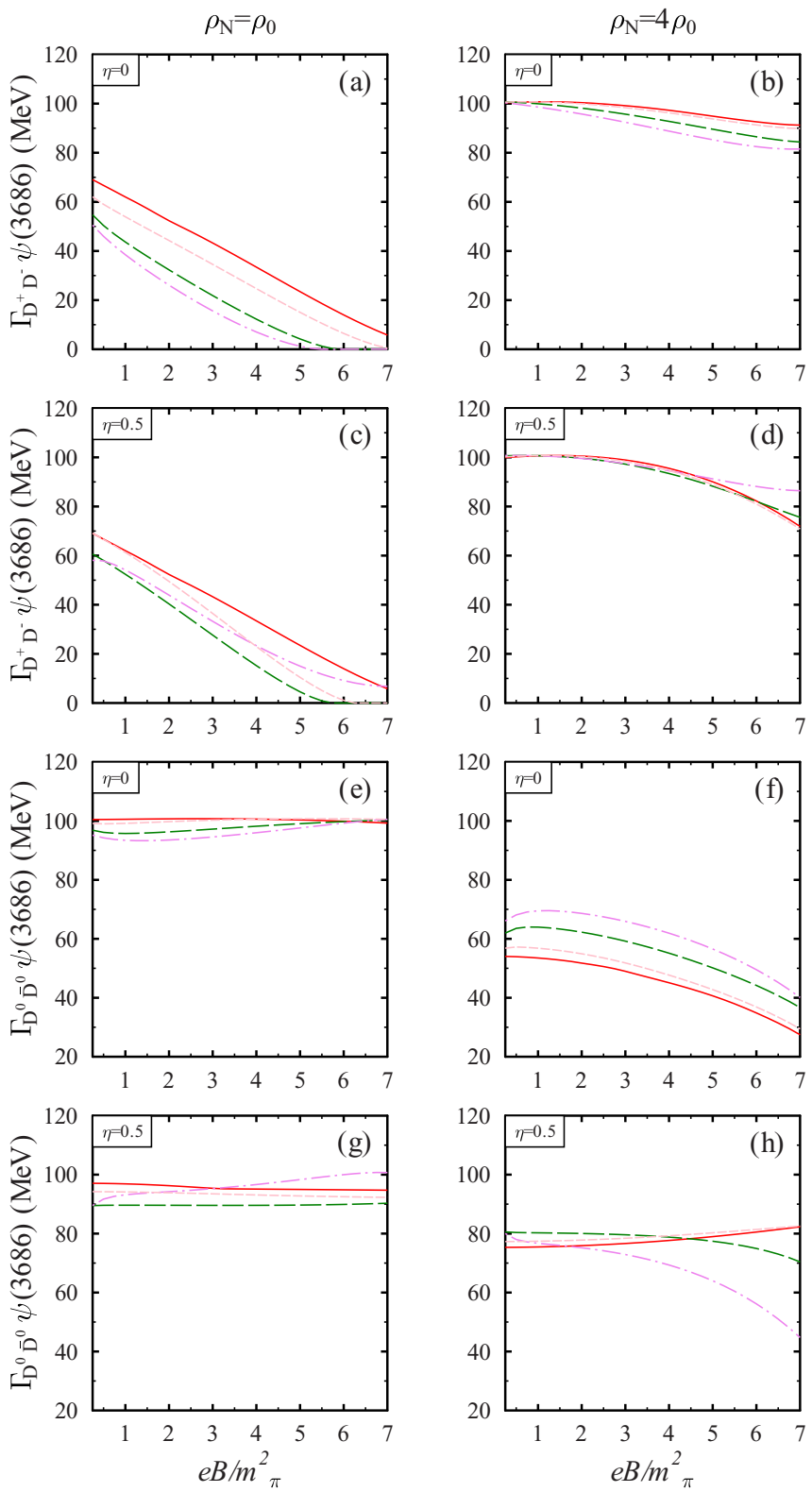

\section{$T=0 \mathrm{MeV}--T=100 \mathrm{MeV}$
$T=50 \mathrm{MeV}-T=150 \mathrm{MeV}$}

FIG. 9. The effective decay width of the charmonium state $\psi(3686)$ decaying into $D \bar{D}$ pairs is plotted as a function of magnetic field $e B$ under different conditions of medium.

decay width of charmonia $\psi(3686)$ and $\psi(3770)$, respectively, decaying into $D \bar{D}$ mesons as a function of magnetic field in the nuclear matter. In Table VI, we have listed the observed values of in-medium decay width of charmonia decaying to $D^{+} D^{-}$and $D^{0} \bar{D}^{0}$ pairs. As one can see from Fig. 9, a nonzero value of decay width of $\psi(3686)$ to $D \bar{D}$ pair is observed because the threshold value of $D \bar{D}$ pair generation is less than the mass of parent meson. For decay channel $\psi(3686) \rightarrow D^{+} D^{-}$, the value of partial decay width of $\psi(3686)$ decreases with the increase in magnetic field and it becomes zero for high values of magnetic field. The zero decay width arises when the threshold value of the $D^{+} D^{-}$pair 
TABLE VI. The values of in-medium decay widths (in MeV) of higher charmonium states $\psi(3686), \psi(3770)$ to $D^{+} D^{-}$and $D^{0} \bar{D}^{0}$ pairs for different conditions of the medium.

\begin{tabular}{|c|c|c|c|c|c|c|c|c|c|}
\hline & \multirow{2}{*}{$e B / m_{\pi}^{2}$} & \multicolumn{4}{|c|}{$\eta=0$} & \multicolumn{4}{|c|}{$\eta=0.5$} \\
\hline & & \multicolumn{2}{|c|}{$T=0$} & \multicolumn{2}{|c|}{$T=100$} & \multicolumn{2}{|c|}{$T=0$} & \multicolumn{2}{|c|}{$T=100$} \\
\hline \multirow[t]{3}{*}{$\Gamma_{D^{+} D^{-}}[\psi(3686)]$} & 0 & 75 & 97 & 59 & 99.9 & 82 & 96 & 69 & 98 \\
\hline & 4 & 33 & 97 & 12 & 93 & 28 & 96 & 15 & 93 \\
\hline & 6 & 14 & 93 & 0 & 86 & 4 & 82 & 0 & 82 \\
\hline & 4 & 74 & 58 & 71 & 63 & 74 & 61 & 71 & 63 \\
\hline & 6 & 71 & 63 & 64 & 67 & 68 & 69 & 63 & 69 \\
\hline \multirow[t]{3}{*}{$\Gamma_{D^{0} \bar{D}^{0}}[\psi(3686)]$} & 0 & 100 & 47 & 94 & 58 & 95 & 71 & 86 & 76 \\
\hline & 4 & 101 & 45 & 98 & 55 & 95 & 78 & 90 & 79 \\
\hline & 6 & 100 & 35 & 100 & 44 & 95 & 81 & 90 & 75 \\
\hline$\Gamma_{D^{0} \bar{D}^{0}}[\psi(3770)]$ & 0 & 51 & 1.3 & 62 & 4.6 & 60 & 10 & 67 & 14 \\
\hline
\end{tabular}

becomes more than the parent meson's mass. The decrement in decay width is due to the calculation of partial decay width [see Eq. (52)] which depends on the energy $E_{D^{+} / D^{-}}$that further depends on the in-medium mass $m_{D^{+} / D^{-}}^{* *}$. As discussed, in the present work, we have taken averaged meson current for $D^{+}$and $D^{-}$mesons [see Eq. (20)] and hence the effective masses of $D^{+}$and $D^{-}$mesons will be the same (and similarly for scalar $D^{0}$ and $\bar{D}^{0}$ ) [24,28,63]. Since the effective mass of charged mesons increases in magnetic field, this will lead to an increase of threshold value of $D^{+} D^{-}$pairs and hence a drop in dcay width. From Fig. 5, we can see that the mass of $D^{+}$mesons (threshold value of $D^{+} D^{-}$pairs) decreases with the increase in density, whereas it increases with the increase in temperature. This explains why the partial decay width of $\psi(3686)$ increases with the increase in density, whereas it decreases as a function of temperature.

When we move from isospin-symmetric medium to asymmetric medium, we observed appreciable effects for density and magnetic field, whereas the least effects are shown as a function of temperature. This is the reflection of the observation of the in-medium mass of daughter meson in the respective medium, which occurs due to the unbalanced behavior of the scalar density of neutrons and protons under magnetic effects [74]. In the decay channel $\psi(3686) \rightarrow$ $D^{0} \bar{D}^{0}$, in Fig. 5(c) [Fig. 5(g)] at $\eta=0(0.5)$, we observe that the decay width remains almost the same (decrease) for low values of temperature, whereas for higher values of temperature it increase as a function of magnetic field. Note that the trend of decay width of decay channel $\psi(3686) \rightarrow D^{0} \bar{D}^{0}$ with magnetic field is opposite to decay channel $\psi(3686) \rightarrow$ $D^{+} D^{-}$. This is because as $D^{0}$ and $\bar{D}^{0}$ are neutral charged mesons, they do not exhibit Landau quantization and hence mass does not increase but decreases as a function of magnetic field as discussed in the last subsection in detail.

In the high-density regime, at $\eta=0$, the partial decay width of the parent meson decreases with the increase in magnetic field and increases as a function of temperature.
However, for $\eta=0.5$, it increases for lower values of temperature and decreases for higher values of temperature. These observations are contradictory to the explanation given in the previous paragraph as the high decrease in the mass causes a decrease in decay width, too. In Eq. (52), the decay width is the product of Gaussian and polynomial expression. In high density, the polynomial part of the decay width dominates the Gaussian part. In Table VI, we have also compared the results with and without magnetic effects. We see that the increase in magnetic field causes more decrease in the value of decay width in low density as compared to high density for the $D^{+} D^{-}$decay channel, and for a neutral $D$ meson pair, the decay width increases for low density and decreases for high density.

In Fig. 10, we plot the results of decay width for decay channels $\psi(3770) \rightarrow D^{+} D^{-}$and $\psi(3770) \rightarrow D^{0} \bar{D}^{0}$ for the same parameters. For the former case, at lower density and particular value of temperature, the decay probability decreases slowly with the increase in magnetic field. The temperature and asymmetric effects are appreciable in the high magnetic-field regime. At high density, the trend of decay width is exactly opposite as a function of magnetic field and temperature. The values of decay width increase with the increase in magnetic field which is due to the higher mass of parent meson $\psi(3770)$. The mass of parent meson rectifies the center-of-mass momentum $p_{D}$, which results in the modification of the Gaussian expression. The interplay between Gaussian and polynomial expression leads to the above observations. In the same figure, for the decay channel $\psi(3770) \rightarrow D^{0} \bar{D}^{0}$, at $\eta=0$, the decay width decreases with increasing magnetic field for high as well as low density. However, for high density the decay probability is much lower due to the presence of the Gaussian term $\left[e^{-\frac{y^{2}}{2\left(1+2 r^{2}\right)}}\right]$ in Eq. (53) and the larger drop in mass of the neutral $D^{0}$ meson. In highly asymmetric matter, the value of the decay probability increases for low temperature and decreases for higher temperature as a function of magnetic field. This result 

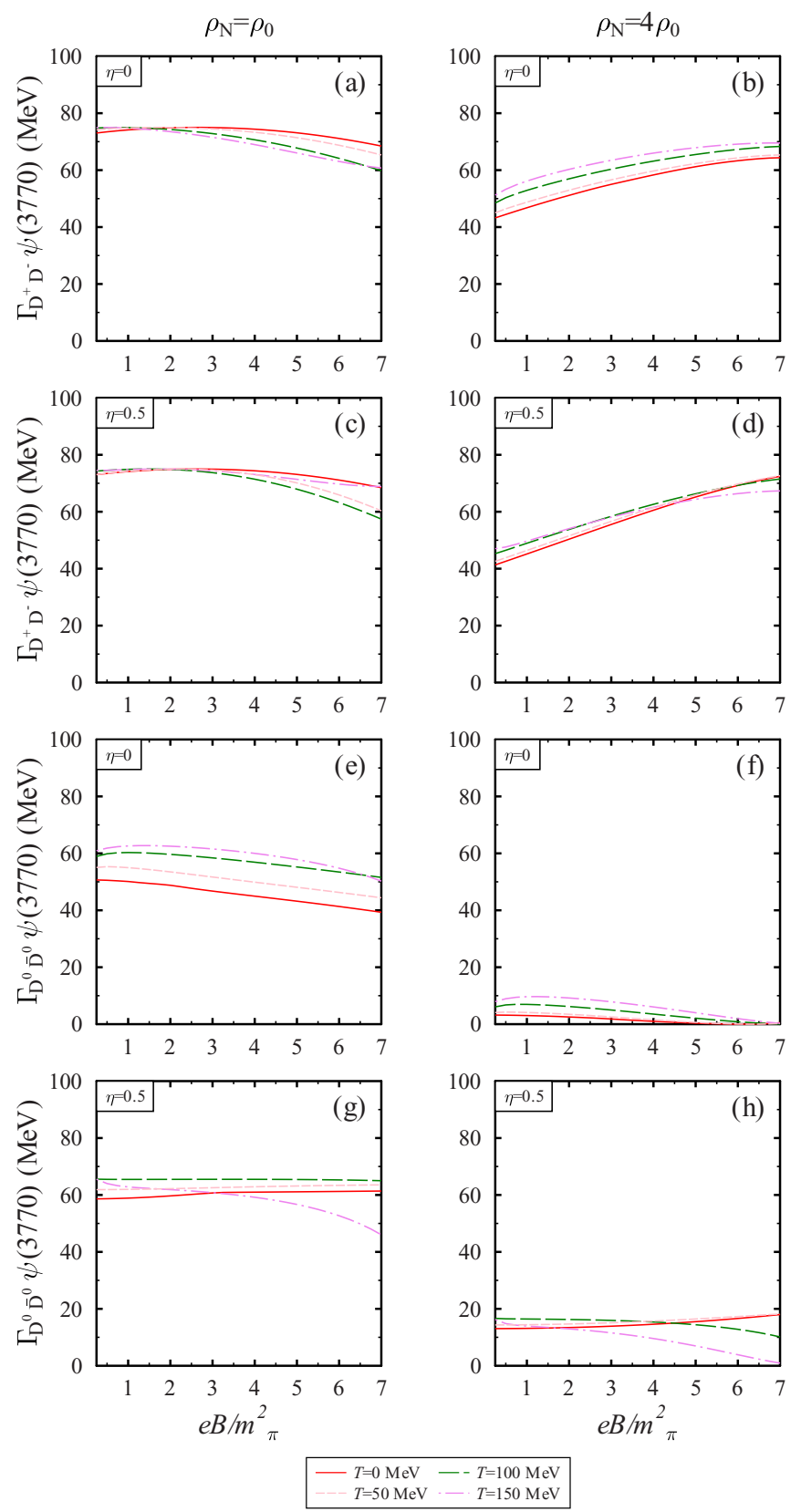

FIG. 10. The effective decay width of the charmonium state $\psi(3770)$ decaying into $D \bar{D}$ pairs is plotted as a function of magnetic field $e B$ under different conditions of medium.

is a reflection of the variation of in-medium mass in asymmetric magnetized nuclear matter. With the comparison of decay width of $\psi(3770) \rightarrow D^{+} D^{-}$in the absence of magnetic field, one can see that the value of decay width at a particular combination of medium parameters increases in the magnetic field due to Landau levels and Gaussian interaction, whereas it decreases for the $D^{0} \bar{D}^{0}$ case.

We have also calculated the decay widths of excited charmonium states $\chi_{c_{0}}$ and $\chi_{c_{2}}$. The vacuum mass of $\chi_{c_{0}}$ and $\chi_{c_{2}}$ is less than the threshold value for the decay products $(D \bar{D})$ and therefore the decay $\chi(3414) \rightarrow D \bar{D}$ and $\chi(3556) \rightarrow D \bar{D}$ is not possible. However, if the mass of the $D$ meson drops appreciably and the threshold value become less than the mass of the parent meson, then decay is possible. We have observed zero decay probability for the $\chi(3414)$ mesons in $D^{+} D^{-}$pairs at all conditions of the medium. But for decay product $D^{0} \bar{D}^{0}$, we have observed finite decay probability in the high-density regime for the nonmagnetic case. For example, in nonmagnetic cold nuclear matter at $\eta=0$ and $\rho_{N}=\rho_{0}\left(4 \rho_{0}\right)$, the value of the decay width of $\chi(3414)$ is 0 (5.6) MeV. Moreover, for decay channel $\chi(3556) \rightarrow D \bar{D}$, we have observed a small finite decay width for low values of magnetic field up to $e B / m_{\pi}^{2}=2$ at high density only. For instance, at $\eta=0, T=0$, and $4 \rho_{0}$, we observed the values of decay width of $\chi$ (3556) to be 124 (158) $\mathrm{MeV}$ at $e B / m_{\pi}^{2}=$ 4 (6). The values of decay width decrease with the increase in temperature but the trend with respect to the magnetic field remains same, whereas for asymmetric matter it decreases for lower temperature and increases for higher temperature with respect to the magnetic field. For example, at $\eta=0.5$, $\rho_{N}=4 \rho_{0}$, and $T=0 \mathrm{MeV}$, the value of the decay width is 41 (35) $\mathrm{MeV}$ at $e B / m_{\pi}^{2}=4(6)$, whereas at $T=100 \mathrm{MeV}$ the value of the decay width changes to 39 (47) MeV. The value of the decay width of both $\chi$ mesons increases in the symmetric magnetic nuclear matter as compared to zero magnetic-field data, whereas for asymmetric nuclear matter it shows asymmetric variation due to $\delta$-field corrections.

In Ref. [67], the magnetic-field-induced decay width of these four charmonium states has been calculated by considering the in-medium masses of the charmonia as well as $D$ and $\bar{D}$ mesons in the combined approach of the chiral SU(3) model and the ${ }^{3} P_{0}$ model at zero temperature only, whereas as discussed above in detail, our calculations are done at finite temperature which is important for heavy-ion collisions.

\section{CONCLUSIONS}

In the present investigation, we calculated the modification in the in-medium masses and decay constants of pseudoscalar and scalar $D$ mesons under the effect of external magnetic field at finite temperature, asymmetry, and density of the nuclear medium. To calculate the in-medium mass, we used the combined approach of QCD sum rules and the chiral SU(3) model. In nuclear matter, the external magnetic field interacts with charged proton and uncharged neutron (due to nonzero anomalous magnetic moment) which results in the modification of scalar and vector densities of nucleons. This magnetic-field-induced density is used to evaluate the coupled scalar fields of a chiral model which are further used to calculate the medium modified scalar quark and gluon condensates [74]. We found prominent effects of magnetic field on the charged $D^{+}$and $D_{0}^{+}$mesons, whereas for neutral $D$ mesons, the effects are smaller. We found a negative (positive) mass shift for pseudoscalar (scalar) $D$ mesons. The temperature and density effects are also quite appreciable. The intervention of magnetic field depletes the effect of isospin asymmetry effects for the charged one but for uncharged mesons it was quite appreciable with crossover effects. As an application part, we calculated the in-medium decay width of higher charmonia in the ${ }^{3} P_{0}$ model. In this calculation, we neglected the mass modification of parent charmonia. We observed appreciable 
changes in the decay widths of $\psi(3686)$ and $\psi(3770)$ but less modification in $\chi(3414)$ and $\chi(3556)$. The calculated decay width may suppress the $J / \psi$ production and hence may decrease its yield. The experimental verification of obtained results can be done in facilities such as CBM, PANDA, J-PARC, and NICA.

\section{ACKNOWLEDGMENT}

R.K. sincerely acknowledges support from Ministry of Science and Human Resources Development (MHRD), Government of India, via Institute fellowship under the National Institute of Technology Jalandhar.
[1] R. Rapp et al., Prog. Part. Nucl. Phys. 65, 209 (2010).

[2] R. Vogt, Ultra-relativistic Heavy-Ion Collisions (Elsevier, Amsterdam 2007).

[3] D. E. Kharzeev et al., Nucl. Phys. A 803, 227 (2008).

[4] K. Fukushima et al., Phys. Rev. D 78, 074033 (2008).

[5] V. V. Skovov et al., Int. J. Mod. Phys. A 24, 5925 (2009).

[6] D. Kharzeev et al., Strongly Interacting Matter in Magnetic Fields (Springer, Berlin, 2013).

[7] A. Vilenkin, Phys. Rev. D 22, 3080 (1980).

[8] Y. Burnier, D. E. Kharzeev, J. Liao, and H. U. Yee, Phys. Rev. Lett. 107, 052303 (2011).

[9] R. Kumar and A. Kumar, Eur. Phys. J. C 79, 403 (2019).

[10] K. Tuchin, Phys. Rev. C 83, 017901 (2011).

[11] K. Tuchin, Phys. Rev. C 82, 034904 (2010).

[12] K. Tuchin, Phys. Rev. C 88, 024911 (2013).

[13] K. Marasinghe and K. Tuchin, Phys. Rev. C 84, 044908 (2011).

[14] A. Das, S. S. Dave, P. S. Saumia, and A. M. Srivastava, Phys. Rev. C 96, 034902 (2017).

[15] S. Reddy P., Amal Jahan C. S., N. Dhale, A. Mishra, and J. Schaffner-Bielich, Phys. Rev. C 97, 065208 (2018).

[16] S. Cho et al., Phys. Rev. D 91, 045025 (2015).

[17] J. D. Bjorken, Fermilab-Pub-82/59-THY, Batavia (1982).

[18] A. Capella, Phys. Lett. B 364, 175 (1995).

[19] S. Soff et al., Phys. Lett. B 471, 89 (1999).

[20] N. Masera (HELIOS-3 Collaboration), Nucl. Phys. A 590, 93c (1995).

[21] W. K. Wilson et al. (DLS Collaboration), Phys. Rev. C 57, 1865 (1998).

[22] D. K. Srivastava and R. Chatterjee, Phys. Rev. C 80, 054914 (2009).

[23] T. Matsui and H. Satz, Phys. Lett. B 178, 416 (1986).

[24] R. Chhabra and A. Kumar, Phys. Rev. C 98, 025205 (2018).

[25] C. Gerschel and J. Hufner, Phys. Lett. B 207, 253 (1988).

[26] B. Zhang, C. M. Ko, B. A. Li, Z. Lin, and B. H. Sa, Phys. Rev. C 62, 054905 (2000).

[27] G. M. Garcia (ALICE Collaboration), J. Phys. G: Nucl. Part. Phys. 38, 124034 (2011).

[28] R. Chhabra and A. Kumar, Eur. Phys. J. A 53, 105 (2017).

[29] G. Inghirami et al., Eur. Phys. J. C 79, 52 (2019).

[30] B. Friman et al., Phys. Lett. B 548, 153 (2002).

[31] P. Papazoglou, D. Zschiesche, S. Schramm, J. SchaffnerBielich, H. Stocker, and W. Greiner, Phys. Rev. C 59, 411 (1999).

[32] J. D. Walecka, Ann. Phys. 83, 491 (1974).

[33] Y. Nambu and G. Jona-Lasinio, Phys. Rev. 122, 345 (1961).

[34] A. Mishra, E. L. Bratkovskaya, J. Schaffner-Bielich, S. Schramm, and H. Stocker, Phys. Rev. C 69, 015202 (2004).

[35] A. Mishra et al., Eur. Phys. J. A 41, 205 (2009).

[36] A. Kumar and A. Mishra, Phys. Rev. C 82, 045207 (2010).

[37] L. J. Reinders et al., Nucl. Phys. B 186, 109 (1981).

[38] A. Hayashigaki, Phys. Lett. B 487, 96 (2000).
[39] T. Hilger, R. Thomas, and B. Kampfer, Phys. Rev. C 79, 025202 (2009).

[40] L. J. Reinders et al., Phys. Rep. 127, 1 (1985).

[41] F. Klingl et al., Nucl. Phys. A 624, 527 (1997).

[42] F. Klingl et al., Phys. Rev. Lett. 82, 3396 (1999).

[43] P. A. M. Guichon, Phys. Lett. B 200, 235 (1988).

[44] S. W. Hong and B. K. Jennings, Phys. Rev. C 64, 038203 (2001).

[45] K. Tsushima, D. H. Lu, A. W. Thomas, K. Saito, and R. H. Landau, Phys. Rev. C 59, 2824 (1999).

[46] A. Sibirtsev et al., Eur. Phys. J. A 6, 351 (1999).

[47] K. Saito and A. W. Thomas, Phys. Lett. B 327, 9 (1994).

[48] P. K. Panda, A. Mishra, J. M. Eisenberg, and W. Greiner, Phys. Rev. C 56, 3134 (1997).

[49] L. Tolós et al., Phys. Rev. C 70, 025203 (2004).

[50] L. Tolós et al., Phys. Lett. B 635, 85 (2006).

[51] L. Tolós, A. Ramos, and T. Mizutani, Phys. Rev. C 77, 015207 (2008).

[52] J. Hofmann and M. F. M. Lutz, Nucl. Phys. A 763, 90 (2005)

[53] S. Chatterjee and K. A. Mohan, Phys. Rev. D 85, 074018 (2012).

[54] B. J. Schaefer, M. Wagner, and J. Wambach, Phys. Rev. D 81, 074013 (2010).

[55] K. Fukushima, Phys. Lett. B 591, 277 (2004).

[56] K. Kashiwa et al., Phys. Lett. B 662, 26 (2008)

[57] S. K. Ghosh, S. Raha, R. Ray, K. Saha, and S. Upadhaya, Phys. Rev. D 91, 054005 (2015).

[58] T. K. Herbst et al., Phys. Lett. B 731, 248 (2014).

[59] M. Drews et al., Phys. Rev. D 88, 096011 (2013).

[60] S. Furui and A. Faessler, Nucl. Phys. Phys. A 468, 669 (1987).

[61] R. Bonnaz and B. Silvestre-Brac, Few-Body Syst. 27, 163 (1999).

[62] R. Kokoski and N. Isgur, Phys. Rev. D 35, 907 (1987).

[63] Z. Wang, Phys. Rev. C 92, 065205 (2015).

[64] Z. G. Wang and T. Huang, Phys. Rev. C 84, 048201 (2011).

[65] A. Kumar, Adv. High Energy Phys. 2014, 549726 (2014).

[66] P. Gubler et al., Phys. Rev. D 93, 054026 (2016).

[67] A. Mishra et al., Eur. Phys. J. A 55, 99 (2019).

[68] R. Gatto and M. Ruggieri, Phys. Rev. D 83, 034016 (2011).

[69] G. S. Bali, et al., J. High Energy Phys. 02 (2012) 44.

[70] H. Liu et al., Phys. Rev. D 91, 014017 (2015).

[71] C. S. Machado, R. D. Matheus, S. I. Finazzo, and J. Noronha, Phys. Rev. D 89, 074027 (2014).

[72] N. Dhale, S. Reddy P., A. Jahan C. S., and A. Mishra, Phys. Rev. C 98, 015202 (2018).

[73] S. Cho et al., Phys. Rev. Lett. 113, 172301 (2014).

[74] R. Kumar and A. Kumar, Chinese Phys. C 43, 124109 (2019).

[75] A. Jahan et al., arXiv:1807.0757 [nucl-th].

[76] K. Tsushima and F. C. Khanna, Phys. Lett. B 552, 138 (2003).

[77] A. Kumar and A. Mishra, Eur. Phys. J. A 47, 164 (2011). 
[78] A. Kumar and R. Chhabra, Phys. Rev. C 92, 035208 (2015).

[79] R. Bruschini and P. Gonzalez, Phys. Rev. C 99, 045205 (2019).

[80] S. Weinberg, Phys. Rev. 166, 1568 (1968).

[81] S. Coleman et al., Phys. Rev. 177, 2239 (1969).

[82] W. A. Bardeen and B. W. Lee, Phys. Rev. 177, 2389 (1969).

[83] D. Zschiesche, Description of Hot, Dense, and Strange Hadronic Matter in a Chiral $\mathrm{SU}(3)_{L} \times \mathrm{SU}(3)_{R} \sigma$-Model, Diploma thesis, Goethe University Frankfurt, 1997.

[84] A. Broderick et al., Astrophys. J. 537, 351 (2000).

[85] A. E. Broderick et al., Phys. Lett. B 531, 167 (2002).

[86] K. Suzuki et al., Phys. Rev. C 93, 045209 (2016).

[87] V. L. Eletsky, Phys. Lett. B 352, 440 (1995).

[88] E. G. Drukarev and E. M. Levin, Prog. Part. Nucl. Phys. 27, 77 (1991).

[89] R. Thomas et al., Nucl. Phys. A 795, 19 (2007).

[90] L. Micu, Nucl. Phys. B 10, 521 (1969).

[91] A. Le Yaouanc et al., Phys. Lett. B 71, 397 (1977).
[92] A. Le Yaouanc et al., Phys. Rev. D 8, 2223 (1973).

[93] A. Kumar and A. Mishra, Phys. Rev. C 81, 065204 (2010).

[94] E. Eichten, K. Gottfried, T. Kinoshita, K. D. Lane, and T. M. Yan, Phys. Rev. D 17, 3090 (1978).

[95] J. Vijande et al., J. Phys. G: Nucl. Part. Phys. 31, 481 (2005).

[96] K. Saito et al., Mod. Phys. Lett. A 13, 769 (1998).

[97] N. Kaiser, P. de Homont, and W. Weise, Phys. Rev. C 77, 025204 (2008).

[98] G. Q. Li and C. M. Ko, Phys. Lett. B 338, 118 (1994).

[99] A. Gadea, G. de Angelis, C. Fahlander, M. DePoli, E. Farnea, Y. Li, D. R. Napoli, Q. Pan, P. Spolaore, D. Bazzacco, S. M. Lenzi, S. Lunardi, C. M. Petrache, F. Brandolini, P. Pavan, C. R. Alvarez, M. Sferrazza, P. G. Bizzeti, A. M. Sona, J. Nyberg, M. Lipoglavsek, J. Persson, J. Cederkall, D. Seweryniak, A. Johnson, H. Grawe, F. Soramel, M. Ogawa, A. Makishima, R. Schubart, and S. Frauendorf, Phys. Rev. C 55, R1(R) (1997). 